Review

\title{
The mitochondrial dynamics in cancer and immune-surveillance
}

\author{
Luca Simula $^{\mathrm{a}, \mathrm{b}}$, Francesca Nazio ${ }^{\mathrm{b}}$, Silvia Campello ${ }^{\mathrm{a}, \mathrm{c}, *}$ \\ a Department of Biology, University of Rome Tor Vergata, Italy \\ b Department of Pediatric Hematology and Oncology, IRCCS Bambino Gesù Children's Hospital, Rome, Italy \\ c IRCCS, Fondazione Santa Lucia, Rome, Italy
}

\section{A R T I C L E I N F O}

\section{Keywords:}

Mitochondrial dynamics

Cancer

Immune-surveillance

Apoptosis

Cell migration

\begin{abstract}
A B S T R A C T
Mitochondria-shaping proteins control the dynamic equilibrium between fusion and fission of the mitochondrial network. Their balance is strictly required to regulate various processes, including the quality of mitochondria, cell metabolism, cell death, proliferation and cell migration. Alterations in these processes are frequently encountered in cancer, during both its onset and later progression, as evidence emerge connecting alterations in mitochondrial dynamics with cancer development. In recent years, novel therapeutic approaches to fight against different human tumors aim at exploiting the immune system's ability to specifically recognize tumor antigens, thus killing malignant cells in a process named immune-surveillance. Interestingly, data are accumulating on the role that mitochondrial dynamics play also for the correct function of both the innate and the adaptive immune system. By this review, we overview how mitochondrial dynamics can affect various processes during cancer development, acting directly on tumor cells or indirectly on cells responsible for tumor aggression and defence.
\end{abstract}

\section{Introduction}

Inside eukaryotic cells, mitochondria exist in a dynamic state, continuously and alternatively undergoing fusion and fission of the network [1]. An interconnected and fused network sustains oxidative phosphorylation (OXPHOS), ATP production, and the mixing of mitochondrial DNA (mtDNA), whilst a partial mitochondria fragmentation tends to isolate damaged portions, thus limiting the stress eventually deriving from a prolonged organelle damage. Fusion of the outer mitochondrial membranes (OMMs) depends mainly on the activity of GTPase Mitofusin proteins (Mfn-1 and -2) [2-4]. These proteins have broad overlapping roles and are expressed in different tissues, with pleiotropic functions. Mfn-2 for example, besides regulating mitochondrial fusion, also directly affects the expression of OXPHOS complex subunits [5], including coenzyme-Q [6], and regulates ERmitochondria contact sites [7]. Fusion of the inner mitochondria membranes (IMMs), instead, is mainly regulated by the dynamin-related GTPase Optic-Athropy-1 (Opa-1) protein [8], normally present in two forms: Opa-1-L, which promotes fusion of IMM, and Opa-1-S, a soluble form, obtained by Opa-1-L proteolytic cleavage, and unable to mediate fusion [9]. Oligomerization of Opa-1, together with ATP synthase subunits $e$ and $g$ [10], Mitofilin and possibly every component of the "mitochondrial contact site and cristae organizing system" (MICOS) complex [11], also regulates the width of cristae junctions, connectionsites between the IMM and the cristae, where OXPHOS complexes and
cytocrome-C (cytC) are localized. Thus, Opa-1 controls cytC release from the cristae during apoptosis (see Section 2.1 for details) $[12,13]$. Moreover, Opa-1 is a regulator of the mitochondrial quality control. Indeed, reduction in mitochondrial membrane potential $(\Delta y)$ stimulates an imbalance toward the Opa-1-S isoform, thus inhibiting mitochondrial fusion and allowing damaged mitochondria to be degraded through mitophagy [14]. Fusion of the mitochondrial network is also very important for regulating the mixing of mtDNA among these organelles [15]. In humans, mutations in fusion proteins are associated with neuropathies, such as Charcot-Mary-Tooth type-2A disease [16] and Dominant-Optic-Atrophy [17]. Moreover, MSTO1 has been recently identified as an additional regulator of the mitochondria fusion, the first cytoplasmic player of the fusion machinery found. Interestingly, its mutation is associated with development of myopathy, ataxia and neurodevelopmental impairment in humans [18].

Mitochondrial fission is important for various cellular processes, such as mitochondrial clearance through mitophagy [19], ROS production [20], regulation of cell proliferation [21-23] and apoptosis $[24,25]$. The main protein driving mitochondrial fragmentation is the GTPase Dynamin-Related-Protein-1 (Drp1) [26]. Mechanistically, the endoplasmic reticulum (ER) and actin filaments can generate an initial constriction at OMM $[27,28]$, which subsequently allows for Drp1 recruitment and aggregation into oligomeric ring-like structures at the point of future fission events [29], where it wraps and severs mitochondrial membranes through its GTP hydrolysis [30,31]. Drp1 is

\footnotetext{
* Corresponding author at: Department of Biology, University of Rome Tor Vergata, Via della Ricerca Scientifica, 00133 Rome, Italy.

E-mail address: silvia.campello@uniroma2.it (S. Campello).
} 
post-transcriptionally regulated by phosphorylation, SUMOylation, ubiquitination, S-nitrosylation and O-GlcNAcylation (see [30] for a review). Genetic ablation of Drp1 is embryonically lethal in mice because of abnormal neuronal development [32]. Drp1 does not act alone, but different "accomplices" assist Drp1-docking at OMMs: Fis1, the role of which is still controversial in mammals [33,34]; Mff [35,36]; MiD51 and MiD49 [37]. In addition, other "less conventional" proteins can mediate Drp1 recruitment at OMM, and promote mitochondria fission, such as the cytoplasmic kinase leucine-rich-repeat kinase 2 (LRRK2) $[38,39]$, the membrane trafficking and apoptosis regulator small Ras family member G-protein Rab32 [40,41] and the very recently discovered dynamin-2 (Dyn-2) [42]. Moreover, silencing of the MICOS protein Mitofilin induces the formation of giant and enlarged mitochondria by downregulating the expression of several fusion and fission mitochondria-shaping proteins [43], thus indicating a role for this protein on the correct homeostasis of the mitochondrial morphology.

\section{Mitochondrial dynamics in cancer}

Tumoral cells are characterized by the multistep acquisition of a series of specific hallmarks that ultimately lead to malignant transformation [44]. Among them the most represented are resistance to cell death, acquisition of uncontrolled proliferation and replicative immortality, metastatization and increased invasiveness, dysregulated cell metabolism and ROS production, mitochondrial quality control through mitophagy, together with tissue vascularization. In addition, a tumormicroenvironment (TME) that promotes a tumor cell escape from immune-mediated cellular destruction is created. Interestingly, mitochondrial dynamics are clearly implicated in all these hallmarks/ processes, both in physiological conditions and during neoplastic transformation. In the following paragraphs, we will review this dual (patho-)physiological contribution of mitochondrial dynamics for each process. Moreover, since alterations in mitochondrial-shaping proteins are frequently found in different human cancers, we will also discuss their potential targeting for therapeutical purposes.

\subsection{Mitochondria-shaping proteins in apoptosis}

Mitochondria-dependent apoptosis is the main signalling pathway activated when normal cells undergo non-physiological modifications and must be eliminated. In response to cell stress or damage, the activation of pro-apoptotic family members of the Bcl-2 family, such as Bax, Bak, Bad and tBid, on OMMs triggers the release of cytC and other apoptotic factors through increased OMM permeabilization, thus leading to cell death through caspase activation. Fragmentation of the mitochondrial network is often observed during this process, in different cell types (see [24] for a review). During apoptosis, tBid promotes Bak/Bax proteins colocalization with Drp1 on OMM endoplasmic reticulum (ER)-mitochondria contact sites $[26,45,46]$. Then, by interacting with cardiolipin, Drp1 mediates a hemi-fission of the OMM that facilitates tethering and oligomerization of Bax/Bak proteins [47]. This, in turn, mediates cytC release. Interestingly, Drp1 KO MEFs are still able to undergo apoptosis, although at a slower rate [32], indicating that mitochondrial fragmentation is not absolutely required for apoptosis execution but strongly influences its rate. In addition, while in healthy cells soluble Bax can promote mitochondrial fusion by favouring Mfn-2 complex assembly [48], membrane-bound Bax can inhibit Mfn-2 activity during apoptosis, thus favouring, instead, Drp1driven mitochondrial hemi-fission. The prevention of mitochondrial fission has normally an anti-apoptotic effect: Bcl- $\mathrm{X}_{\mathrm{L}}$ performs part of its anti-apoptotic activity by inhibiting Drp1 [49], and the down-regulation of pro-fission Mff has a considerable anti-apoptotic effect [36]. However, Mfn-1 KO MEFs are resistant to apoptotic stimuli. This apparent contradiction is due to the consequent hyperfragmented phenotype of mitochondria in these cells, which does not allow Bax accumulation into OMMs because of an incorrect OMM curvature of the hyperfragmented organelles [50]. These data suggest that an optimal OMM curvature, induced by partial hemi-fission more than complete mitochondrial fragmentation, is essential to promote cytC release.

Since it is normally stored in the cristae, cytC is released in the cytosol, through permeabilized OMM, upon a first release from the cristae into the inter-membrane space (IMS). This step is regulated, as mentioned above, by the combined activity of MICOS proteins (see [11] for a review) and the correct composition and balance between Opa-1-L and Opa-1-S hetero-oligomers [12], which normally act as a cristaejunction cap that, under mechanical/pro-apoptotic stress conditions, is disrupted and allows the apoptotic factors release. Indeed, ectopic expression of stable Opa-1-L oligomers can prevent cytC release from mitochondria downstream of Bax/Bak activation [51]. Similarly, it has been reported that mitochondrial prohibitin promotes Opa-1 cleavage, and the accumulation of cleaved Opa-1-S isoform in prohibitin KO MEFs induces apoptotic cell death, which is specifically due to abnormal cristae morphology [52].

On the other hand, one of the best-characterized hallmarks of cancer cells is their capability to escape from apoptosis, and several evidence suggest that modulation of mitochondria-shaping protein activity contributes to such phenotype. Pro-oncogenic alterations in MEK/ERK signalling pathway lead to phosphorylation of Mfn-1, increasing its affinity to and sequestering Bax, so preventing apoptosis [53]. In some human colon cancer cell lines, it has been shown that Drp1 favours tumor growth, since its depletion reduces Opa-1-L to Opa-1-S ratio, thereby promoting cytC release from the cristae and apoptosis [54]. By contrast, hypoxic in vitro studies performed in other cell lines from the same tumor highlighted how hypoxic TME can lead to Mfn-1-dependent mitochondria elongation, with consequent increased organelle respiratory capacity and also increased resistance to apoptosis [55]. The resistance to apoptosis upon cisplatin treatment has been associated with i) lung adenocarcinoma patients with an unusual sequestration of Drp1 inside the nucleus (no more available to promote OMM hemifission) [56], ii) in tongue squamous cell carcinoma (TSCC) with the inhibition of Mff protein translation and mitochondria fission [57], and iii) apoptosis-resistant neuroblastoma cells with increased Opa-1 levels and fused mitochondria [58]. Further, deletion of the miR494-containing chromosomal fragment frequently occurs in different cancers [59] and, interestingly, restoring miR494 levels in renal carcinoma cells promotes cellular apoptosis in a process requiring Drp1-dependent mitochondrial fragmentation $[60,61]$. In addition, anticancer drug treatments have been shown to downregulate the activity of different MICOS proteins, thus affecting the cristae shape and presumably inducing oxidative stress $[62,63]$, although the potential involvement of mitochondrial dynamics has been not investigated in these cases. On the contrary, it has been recently demonstrated that during apoptosis the ATP synthase Inhibitory Factor 1 (IF1), which prevents dissipation of ATP levels due to a reversal activity of ATP synthase, is also able to inhibit Oma-1-dependent Opa-1 processing, thus preventing the cristae remodelling and cytocrome-c release [64]. In line with this, IF1 levels correlate with the tumor grade in invasive ductal breast carcinoma, and IF1 KO tumor cells show reduced xenograft tumor growth, suggesting a pro-oncogenic role for IF1 [64].

Therefore, alteration of mitochondrial dynamics are found in different human cancers and are associated with increased resistance to apoptosis. Nevertheless, in a few cases-mostly regarding Drp1, a Janusfaced behaviour for few mitochondria-shaping proteins is reported but not clearly explained yet.

\subsection{Mitochondrial dynamics and cell proliferation}

Another important feature of cancer cells is their ability to undergo continuous proliferation even in the absence of appropriate stimulations. Deregulation of cell cycle arrest checkpoints allows tumor cells to proliferate indefinitely and to acquire a kind of replicative 
"immortality". Mitochondrial fission plays an important role during different phases of physiological cell cycle, and its dysregulation in cancer cells can promote tumor proliferation. Indeed, during mitosis, CDK1/cyclinB-mediated Drp1 phosphorylation at Ser616 is essential for the correct progression of cell division, allowing both daughter cells to receive part of the mitochondrial network [23]. In fact, Drp1-KO MEFs show a reduced proliferation rate, due to impairment on mitochondria redistribution [32]. Moreover, Drp1 down-regulation can also lead to increased ROS production during S-phase, a consequent higher levels of DNA damage, and a longer mitosis length due to accumulation of a hyperfused mitochondrial network around the centrosomes, this impairing their normal function [22].

In the case of neoplastic transformation, large-scale analyses have reported a correlation between Drp1 and cell-cycle genes in 29 different cancer cell types, in which Drp1 positively drives mitotic transition [65]. In addition, Drp1 has been shown to be implicated in the G1-S phase transition in hepatocellular carcinoma (HCC) cells, where increased Drp1 levels promote progression into cell cycle, through p53 inhibition (through ROS-dependent activation of the Akt/MDM2 pathway) and NK-кB activity promotion (which, in turn, induces expression of the specific cyclins D1 and E1) [66]. Further, lung cancer cells show an increased Drp1/Mfn-2 ratio, which promotes mitochondrial fission, whereas genetic manipulations restoring mitochondrial elongation reduce cancer cell proliferation and increase their sensitivity to apoptotic stimuli [67].

In conclusion, Drp1 dysregulation favours cancer cell proliferation by acting on different phases of the cell cycle. Whether this might be true also for other mitochondria-shaping proteins is currently unknown. For example, instead, even if prohibitin downregulation increases Opa1 degradation in cancer cells and correlates with a reduced cell proliferation [68], this is presumably only a consequence of an increased apoptosis, similarly to what suggested in prohibitin KO MEFs ([52], see Section 2.1 for details).

\subsection{Mitochondrial dynamics and cell migration}

The first study that linked mitochondrial dynamics and cell migration dates back to 2006, when it has been shown a pivotal role for mitochondrial morphology on orchestrating leukocytes migration [69]. Mitochondrial fragmentation promotes and regulates cell chemotaxis, by localizing the organelles at the cell rear-edge to locally "fuel" the cell motor myosin II (see Section 3.2 for details). Based on this work, later on, a crucial role has been attributed to mitochondrial dynamics in modulating metastatization of tumor cells. Cancer cells migrate and invade tissues through a fibroblast-like cell migration, this usually being considered as the main hallmark of metastatization of a tumor. This migration relies on Drp1's activity, too [70]. In invasive breast cancer cells, total levels of Drp1 and its phosphorylated levels on activating residue Ser616 are upregulated, while Mfn-1 is downregulated, compared to non-invasive cells [71]. In this case, mitochondria need to fragment, in a Drp1-dependent way, in order to be transported and accumulate at the cell leading-edge, where they promote local ATP production, essential for lamellipodia formation, cell migration and metastasis formation [71]. The same was shown in non-small cell lung cancer (NSLCC) [72], in epithelial cancer cells [73] and malignant oncocytic thyroid tumors [70]. Interestingly, also TME-derived hypoxia is able to regulate both glioblastoma and breast cancer cell migration by promoting Drp1 transcription and stimulating mitochondrial fission $[74,75]$. In addition, the inhibition of mitochondria transport along the microtubules, obtained by downregulation of the Miro1 dynein motor, also prevents epithelial cancer cell migration [73]. Syntaphilin (SNPH), a protein first discovered in neurons for its ability to dock mitochondria to axon microtubules, similarly suppresses mitochondrial dynamic movements, and therefore cell motility, in tumor cells [76]. Consistent with its role as an onco-suppressor, SNPH is downregulated in several malignancies, such as the epithelial and haematological ones [76]. On the contrary, levels of Miro2, which promotes mitochondria movement along microtubules, are upregulated in the same tumors [76]. Moreover, SNPH downregulation correlates also with poor survival prognosis in colon and lung adenocarcinoma patients [76]. Recently, it has been shown that NF- $\mathrm{kB}$-inducing kinase (NIK) localizes at OMM in both MEFs and cancer cell lines where it interacts with Drp1, by promoting its translocation and activation; this favours fragmented mitochondria redistribution to lamellipodia and sustains high-speed cell migration [77]. Such a role is independent from the downstream mediators of NIK signalling [77]. Therefore, it seems that the metastatic potential of cancer cells often correlates with increased Drp1 or decreased Mfns activities, as shown -respectively, for breast cancer and glioma metastasis [71,74], and gastric cancer cells [71]. Drp1-triggered mitochondrial fission modulating cell migration is detected also in Vascular Smooth Muscle Cells (VSMCs) in response to platelet-derived-growthfactor (PDGF) stimulation [78]. Differently from what has been elucidated so far, Caino and colleagues reported that mitochondria accumulation at the leading-edge of migrating glioma cells is Mfn-1-dependent and requires PI3K inhibition to promote focal adhesion turnover and tumor invasiveness. Interestingly, OXPHOS inhibition prevents such mitochondria redistribution, this indicating that ATP production and oxidative metabolism may play an important role during cell migration [79]. Of note, this study may suggest that in cancer cell migration the relative contribution of mitochondrial fission/ fusion equilibrium is highly context-dependent.

\subsection{Mitochondrial dynamics and cell metabolism}

Mitochondrial dynamics are closely linked to cell metabolism. Thinner and elongated mitochondria are often associated with increased OXPHOS activity and sustained ATP production [80], while a shift toward the glycolytic pathway and the inhibition of TCA cycle associate with fission and more widened cristae structure. Downregulation of Mfns or Opa-1 levels reduces OXPHOS and ATP production in several cell models [81-83]. In line with this, Opa-1-driven cristae remodelling is strictly required for regulating ATP production upon different cell needs [84], while Mfn-2 overexpression in myotubes can upregulate the transcription of OXPHOS genes [85]. However, in HeLa cells, prolonged Drp1 downregulation can reduce respiration levels [86], this suggesting that optimal OXPHOS activity requires a balanced equilibrium in mitochondrial morphology. Very recently, even in induced pluripotent stem cells (iPS), the OXPHOS-to-glycolysis switch has been associated with increased mitochondrial fragmentation due to ERK-dependent Drp1 activation [87]. Not only the modulation of mitochondrial morphology can influence respiration and the bioenergetics of the cell, but also the opposite is true. For example, nutrient withdrawal can promote mitochondrial elongation through either Drp1 inactivation [88] or Mfn-1 stabilization [89]. On the contrary, the nutrient excess in a high-fat diet can reduce Mfn-2 transcription and promote mitochondrial fragmentation [90].

One of the main physiological modifications that tumor cells undergo is their switch from OXPHOS to glycolysis, a process known as Warburg effect [91,92]. This metabolic switch, even in conditions of high-oxygen tension, allows tumor cells to sustain their rapid proliferation rate and to better survive under TME hypoxic conditions. Moreover, glycolysis can supply carbon source for anabolic reactions [93]; lactate, a glycolytic by-product released by tumor cells, can thus be taken up by stromal cells, and converted in pyruvate that is released again in the TME to refuel cancer cells [94]. Mutations in tumor cells affecting mitochondrial respiration, such as in tricarboxylic acid (TCA) cycle enzymes, may reprogram tumor cell metabolism, conferring higher survival and migratory capabilities $[95,96]$.

In sum, the glycolytic switch is an important mediator of cancer growth $[97,98]$. As previously stated, this switch frequently occurs along with fragmentation of the mitochondrial network [22,99]. For example, a correlation between mitochondrial fission and glycolysis is 
observed in Survivin-overexpressing neuroblastoma cells [100] and 3BrPA-treated urinary bladder cancer cells [101]. Among epithelial ovarian cancer (EOC) cell lines, whilst most ovarian clear cell carcinoma (OVCC) cells show high glycolysis and mitochondrial respiration rate, OVCA420 cells mainly rely on glycolysis, showing a low oxygen consumption rate (OCR), and this correlates with fragmentation of mitochondrial network and increased Drp1 expression [102]. Moreover, increased Drp1 expression in OVCA420 cells impairs HIF-1 $\alpha$ upregulation upon hypoxia, thus rendering these cells unable to cope with the stress [102]. Nevertheless, as previously stated, optimal OXPHOS activity has been suggested to play a role in supporting tumor cell invasiveness [79], thus highlighting the metabolic plasticity of cancer cells in different conditions. Despite the numerous examples here reported, correlating an altered cellular metabolism with a modification of the mitochondrial morphology in cancer cells, it is not clear yet whether modulation of the mitochondrial network in cancer cells is a priming event or just a consequence of the OXPHOS-glycolysis metabolic shift. Further, since most of mitochondrial genes encode ETC complex proteins involved in OXPHOS activity, excessive mtDNA mutational load in cancer cells may favour the OXPHOS-glycolysis switch, thus favouring tumorigenesis [103]. Moreover, there is an association with low mtDNA copy number and poor survival rate in kidney and adrenocortical carcinoma patients, indicating that overall mtDNA amounts can be reduced in tumor masses compared to adjacent normal tissue [104]. Mitochondrial dynamics are essential regulators of the mtDNA copy number and a tight fusion/fission balance must be kept to secure mtDNA integrity. Interconnection of the network helps in maintaining a homogeneous mtDNA population among various mitochondria, as confirmed by Mfns downregulation in muscle cells leading to loss of mtDNA copies and accumulation of point mutation in mtDNA, so driving dysfunction in the organelles [15]. On the other side, accumulation of mutated mtDNA copies into fragmented and dysfunctional organelles could promote their degradation through mitophagy (see below), with subsequent mitochondrial biogenesis to recreate a healthy network. Preventing Drp1-dependent sequestration of damaged organelles impairs mitophagy and leads to accumulation of damaged mitochondria, so decreasing respiration capacity and ATP production [105].

In conclusion, there is increasing evidence for a complex relationship among the different metabolic requirements of tumor cells within the TME and the shape of their mitochondria. A deeper understanding of the potential impact in modulating the metabolism of tumor cells (ideally by directly affecting mitochondrial dynamics) for controlling tumor growth is of the highest priority.

\subsection{Mitochondrial dynamics and ROS production in cancer}

Reactive Oxygen Species (ROS) are normal by-products of cellular metabolism. Although in particular conditions ROS can even become part of several signalling pathways [106], their upregulation is frequently harmful to the cells, which have evolved several antioxidant mechanisms to regulate ROS homeostasis (see [107] for a review). Mitochondrial dynamics are physiologically implicated in regulating ROS production. Indeed, the mitochondrial respiratory chain continuously produces physiological levels of ROS, even though these levels can drastically increase upon modifications of the mitochondrial network morphology. ROS increase has been associated with Drp1-dependent mitochondrial fragmentation after ionizing radiation in human fibroblast-like cells [108] and upon genetic overexpression of Drp1; the down-regulation of the same protein, instead, can lead to a drop in mitochondrial ATP production and OXPHOS activity; this, in turn, increases ROS production [22]. Similarly, cells lacking Opa-1 or Mfn-1-2 loose mitochondrial membrane potential $(\Delta \psi)$, reduce their oxygen consumption rate (OCR), and increase ROS production [81,109]; by contrast, genetically shifting the mitochondria balance toward elongation decreases ROS amounts in several experimental models [83].
Finally, accumulated ROS (and reactive nitrogen species, RNS) are able, in turn, to modify either directly or indirectly several mitochondriashaping proteins, thus creating a positive feedback loop $[110,111]$.

Accumulation of ROS amounts in tumor cells is a potent driver of initial cancer development. Although a detailed analysis of ROS effects on tumor growth is beyond the scope of this review (see [112] for details), we would like to stress that ROS accumulation could represent a priming signal during the tumor onset, this being the probable cause of mtDNA mutations often found in cancer cells [112,113]. Elevated ROS can also promote upregulation of several oncogenic signalling pathways, such as Ras/MAPK/ERK, PI3K/Akt and IKK/KF-B, and can favour tumor cell proliferation, apoptosis-resistance and cell migration (see [112] for a review). On the contrary, at later stages of cancer progression, a controlled reduction of ROS amounts in tumor cells promotes tumor growth and metastasis formation, as in melanoma and lung cancer mouse models [114,115], this indicating an important correlation between ROS accumulation and cancer stage. Further, the pro-angiogenesis activity of tumor cells becomes crucial for their survival in a microenvironment progressively more hypoxic, for a continuous supply of nutrients and oxygen [116]. The hypoxic TME triggers, also through oxidative stress - as shown in ovarian and prostate cancers [117-119], the tumor production of angiogenetic factors such as vascular-endothelial-growth-factor (VEGF), by upregulating hypoxia-inducible factor- $1 \alpha$ (HIF-1 $\alpha$ ) [120]. Interestingly, HIF- $1 \alpha$ is able to limit ROS accumulation in tumor cells, thus promoting cancer progression at later stages, by limiting the production of acetyl-CoA (the key molecule entering the TCA cycle) from glycolysis [121] and fattyacid oxidation [122]. Indeed, the activity of TCA cycle enzymes and ETC complexes under hypoxic conditions may lead to uncontrolled ROS accumulation, which is harmful to cancer cells [123].

In conclusion, mitochondrial dynamics has the potential to tightly modulate ROS production inside the TME thus becoming active players in the tumor onset and development. However, despite a well-documented role of ROS in promoting initial cancer onset, their role during later cancer progression is more controversial.

\subsection{Mitochondrial dynamics and mitophagy in cancer}

Damaged mitochondria can be removed from the cell through a selective autophagy process termed mitophagy. In order to maintain overall mitochondria good quality, organelles with decreased membrane potential and respiratory capability must be isolated from the healthy network [124], targeted to clearance and, eventually, engulfed by autophagosome for being transported to lysosomes and there lysed.

The Parkin/PINK1 axis mediates mitophagy signalling: in more details, the kinase PINK1 recruits the ubiquitin-E3 ligase Parkin that ubiquitinates several OMM proteins and creates a positive feedback loop with PINK1 and the mitophagic receptors NDP52 and OPTINEURIN. These receptors mediate the engulfment of targeted mitochondria by autophagosome for lysis [125-127]. Other Parkin/ PINK1-independent pathways can mediate mitophagy alternatively involving NIX/BNIP3, Ambra1, ULK1 or cardiolipin in neurons [128-132]. Interestingly, among the Parkin targets are Mfn-1-2 and Miro1. Parkin-mediated phosphorylation of Mfns promotes their degradation through the proteasome, inhibiting fusion of damaged organelles with the rest of mitochondrial network [133]; the inhibitory phosphorylation of the dynein motor Miro1, instead, blocks mitochondrial motility [134]. Thus, mitochondrial fission is strictly linked to mitophagy, also for obvious space limitation reasons, and it is triggered not only by Mfns inactivation. Indeed, the autophagy-inducer and energy-sensor adenosine monophosphate (AMP)-activated protein kinase (AMPK) can directly phosphorylate the Drp1-receptor Mff, thus promoting mitochondrial fission [135].

Mitophagy is important also in cancer progression. Defective removal of damaged mitochondria could lead to increased ROS amounts with consequent accumulation of mutations in mitochondrial and 
nuclear DNA, thus favouring cancer onset. Mitophagy has been implicated in the removal of damaged mitochondria containing high levels of ROS [136,137] and ROS accumulation can promote by itself mitophagy activation, for example through mitochondrial membrane depolarization and Parkin/PINK1 pathway activation [138,139], thereby inhibiting initial tumor growth. Indeed, Parkin deficient mice spontaneously develop hepatic tumors [140], and PINK1 mutations have been described in neuroblastoma [141]. The same is observed in mice deficient for different key proteins of the more general bulk autophagy, such as Beclin 1, Atg5-7 and Ambra1 in heterozygosity $[142,143]$. Whilst mitophagy impairment could be beneficial for the tumor onset, at later stages of tumor progression mitophagy could become protective for these cells, presumably protecting tumor cells from excessive mitochondrial damage, ROS accumulation and apoptosis. For example, Drp1-dependent mitophagy is an important pro-survival event in hepatocellular carcinoma (HCC), protecting cancer cells from apoptosis through p53 and NF-kB modulation [144]. Similarly, in K-Ras induced lung cancers, inhibition of mitophagy leads to strong reduction in cell proliferation [145].

Therefore, considering both mitophagy double role in cancer onset and progression, and the impact of mitochondrial dynamics in regulating this process, further research into the modulation of these morphological dynamics during different phases of cancer might be crucial for therapeutic purposes.

\subsection{Mitochondrial dynamics as possible therapeutic targets}

By summarizing the main aspects of the involvement of mitochondria dynamics in cancer growth, a picture seems to emerge, in which tumor cells are able to modify the shape of mitochondrial network according to their needs at different cancer stages (Fig. 1). Indeed, mitochondrial fusion can favour tumor cell resistance to apoptosis, while mitochondrial fission has been associated with increased invasiveness and proliferation. Moreover, different requirements regarding cell metabolism, ROS production and mitophagy in cancer progression go along with concomitant modifications of the mitochondrial morphology. Therefore, it is not surprising that specific alterations in mitochondrial dynamics have been found associated with several cancer types, and also with their survival prognosis, thus indicating them as strong candidate targets for future therapeutic approaches.

Imbalance towards fragmentation of the mitochondrial network is often found in several cancer cells [146]. Interestingly, the oncogenic MAPK/ERK pathway, which is a driver of cancer progression in different tumors, is able to upregulate Drp1 activity in different cancers $[147,148]$ by phosphorylation on serine residue Ser616 [147]. In several human cancers, a poor survival rate is associated with an increased Drp1 level [54,67,70,71,144,149] or a decreased Mfn-2 [150,151] or Mfn-1 amounts [144,152], as also confirmed by the emerging Bioinformatics databases displaying the expression levels of different mitochondria-shaping proteins in human tumors (see The Human Atlas Project [153] and Table 1 for details).

Even more interestingly, mitochondrial dynamics seem to be essential mediators of apoptosis-induction by different chemotherapeutic drugs, this heralding the use of their modulation. Indeed, upregulated Drp1 activity is essential for the induction of apoptosis by Benzil isothiocyanate (BITC), Differentiation -Inducing -Factor 3 (DIF-3), Micheliolide (MCL) and CGP37157 [154-157].

The main limitation for considering the mitochondrial dynamics as therapeutic targets in human cancer is the poor availability of specific drugs that can selectively affect the activity of mitochondria-shaping

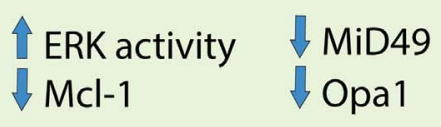

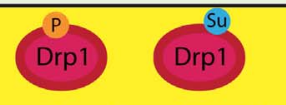

-colocalization with pro-apoptotic factors - OMM permeabilization - Cyt-c release

\section{Cancer cell}

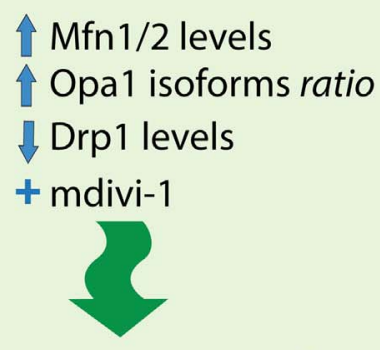

\section{prevented mitochondrial fission}

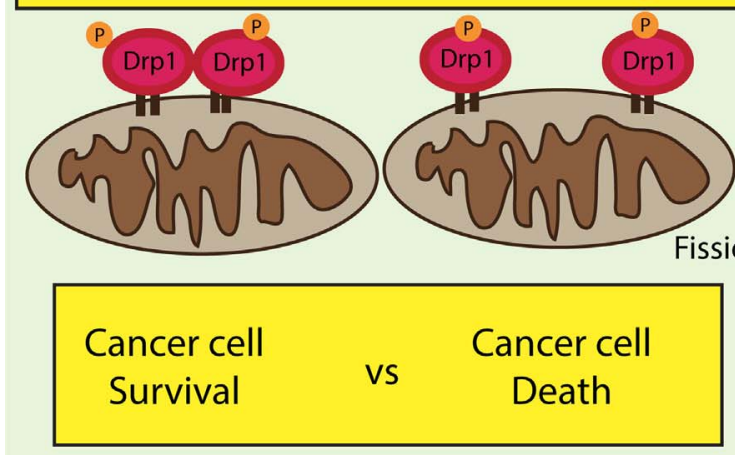

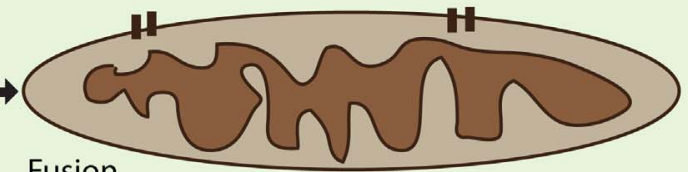

Fusion

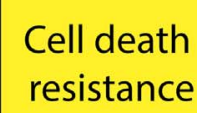

Inhibition of

Vs migration and metastatization

Fig. 1. Different roles of mitochondrial dynamics in cancer cells.

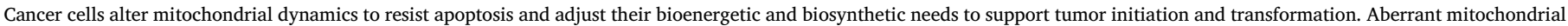

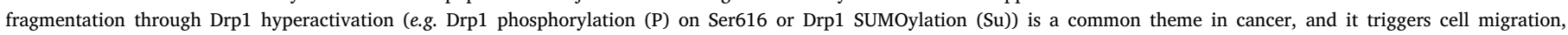

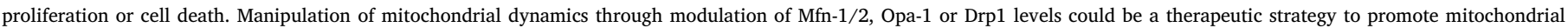
fission and to induce cancer cell death. 
Table 1

Dissection of mitochondria shaping protein expression levels in several tumors, compared to adjacent normal tissue.

\begin{tabular}{|c|c|c|c|c|c|c|c|c|}
\hline & $\mathrm{RE}$ & Drp1 & Fis1 & Mff & MiD49 & Mfn1 & Mfn2 & Opa1 \\
\hline \multirow[t]{2}{*}{ breast cancer } & up & $8 / 24$ & $1 / 11$ & $2 / 12$ & $1 / 12$ & $3 / 11$ & - & $1 / 23$ \\
\hline & down & - & - & $2 / 12$ & - & - & $8 / 11$ & $5 / 23$ \\
\hline \multirow[t]{2}{*}{ carcinoid } & up & $2 / 8$ & $3 / 4$ & - & - & $3 / 3$ & - & $8 / 8$ \\
\hline & down & - & - & $3 / 4$ & $1 / 4$ & - & $3 / 4$ & - \\
\hline \multirow[t]{2}{*}{ cervical cancer } & up & $6 / 24$ & $2 / 12$ & $1 / 12$ & - & $7 / 11$ & - & - \\
\hline & down & - & $6 / 12$ & - & - & $1 / 11$ & - & $1 / 20$ \\
\hline \multirow[t]{2}{*}{ colorectal cancer } & up & - & - & $1 / 11$ & - & - & - & $9 / 24$ \\
\hline & down & - & $3 / 11$ & - & $9 / 10$ & - & $10 / 12$ & $1 / 24$ \\
\hline \multirow[t]{2}{*}{ endometrial cancer } & up & $13 / 23$ & - & - & $2 / 12$ & - & - & $2 / 20$ \\
\hline & down & $2 / 23$ & $8 / 11$ & $2 / 10$ & $8 / 12$ & $2 / 11$ & - & $2 / 20$ \\
\hline \multirow[t]{2}{*}{ glioma } & up & $9 / 21$ & $11 / 11$ & $7 / 12$ & $3 / 11$ & $5 / 9$ & $2 / 11$ & $8 / 22$ \\
\hline & down & $2 / 21$ & - & - & - & - & $5 / 11$ & $10 / 22$ \\
\hline \multirow[t]{2}{*}{ head neck cancer } & up & $1 / 7$ & - & - & - & - & $1 / 4$ & $6 / 8$ \\
\hline & down & - & - & - & - & $1 / 4$ & - & - \\
\hline \multirow[t]{2}{*}{ liver cancer } & up & $19 / 24$ & $5 / 11$ & $6 / 12$ & $3 / 11$ & - & $3 / 12$ & $5 / 24$ \\
\hline & down & $4 / 24$ & - & - & - & $1 / 12$ & - & $1 / 24$ \\
\hline \multirow[t]{2}{*}{ lung cancer } & up & - & - & - & - & - & $1 / 9$ & - \\
\hline & down & $1 / 23$ & $1 / 11$ & - & $8 / 12$ & - & $8 / 9$ & $5 / 18$ \\
\hline \multirow[t]{2}{*}{ lymphoma } & up & $5 / 24$ & $2 / 11$ & $1 / 11$ & $2 / 10$ & $2 / 11$ & - & $1 / 24$ \\
\hline & down & $2 / 24$ & - & $8 / 11$ & - & - & $1 / 11$ & $8 / 24$ \\
\hline \multirow[t]{2}{*}{ melanoma } & up & $24 / 24$ & $9 / 9$ & $2 / 12$ & $6 / 12$ & $3 / 11$ & $1 / 12$ & $17 / 18$ \\
\hline & down & - & - & $2 / 12$ & $3 / 12$ & $3 / 11$ & $10 / 12$ & - \\
\hline \multirow[t]{2}{*}{ pancreatic cancer } & up & $2 / 22$ & $3 / 11$ & - & $3 / 11$ & $10 / 10$ & $5 / 12$ & - \\
\hline & down & $3 / 22$ & - & $2 / 12$ & $5 / 11$ & - & - & $1 / 17$ \\
\hline \multirow[t]{2}{*}{ prostate cancer } & up & $5 / 21$ & $9 / 10$ & - & $1 / 11$ & $1 / 12$ & - & $21 / 24$ \\
\hline & down & - & - & $3 / 12$ & $9 / 11$ & - & $10 / 11$ & - \\
\hline \multirow[t]{2}{*}{ renal cancer } & up & $1 / 23$ & - & - & - & - & - & - \\
\hline & down & $11 / 23$ & $12 / 12$ & $10 / 12$ & $8 / 12$ & $5 / 12$ & $12 / 12$ & $22 / 24$ \\
\hline \multirow[t]{2}{*}{ skin cancer } & up & $3 / 24$ & - & - & - & $6 / 12$ & $1 / 12$ & - \\
\hline & down & $2 / 24$ & $7 / 8$ & $5 / 11$ & $12 / 12$ & $3 / 12$ & - & $7 / 23$ \\
\hline \multirow[t]{2}{*}{ stomach cancer } & up & - & - & - & - & $1 / 12$ & - & - \\
\hline & down & $3 / 22$ & $9 / 10$ & $1 / 11$ & $10 / 10$ & $1 / 12$ & - & $2 / 15$ \\
\hline \multirow[t]{2}{*}{ testis cancer } & up & $9 / 23$ & $2 / 9$ & - & - & - & - & $2 / 22$ \\
\hline & down & $1 / 23$ & $3 / 9$ & $10 / 11$ & $10 / 12$ & $7 / 12$ & $7 / 10$ & $4 / 22$ \\
\hline \multirow[t]{2}{*}{ thyroid cancer } & up & $2 / 8$ & - & - & - & $1 / 4$ & - & $2 / 6$ \\
\hline & down & - & - & - & $1 / 4$ & - & $2 / 4$ & - \\
\hline \multirow[t]{2}{*}{ urothelial cancer } & up & $4 / 22$ & - & - & $1 / 10$ & - & - & - \\
\hline & down & $2 / 22$ & $6 / 11$ & $4 / 12$ & $7 / 10$ & $10 / 11$ & $9 / 11$ & $2 / 20$ \\
\hline \multirow[t]{2}{*}{ all cancers } & up & $113 / 367(30.79 \%)$ & $47 / 173(27.16 \%)$ & 20/177 (11.29\%) & $22 / 176(12.5 \%)$ & 42/168 (25\%) & $14 / 158(8.86 \%)$ & $72 / 360(20 \%)$ \\
\hline & down & 33/367 (8.99\%) & $55 / 173(31.79 \%)$ & $52 / 177(29.37 \%)$ & $91 / 176(51.70 \%)$ & $34 / 168(20.23 \%)$ & $85 / 158(53.79 \%)$ & 71/360 (19.7\%) \\
\hline
\end{tabular}

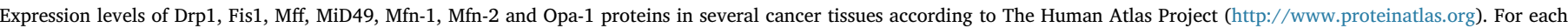

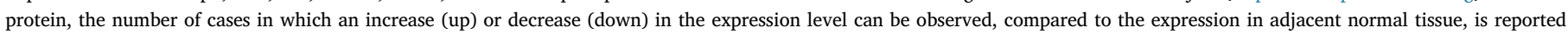

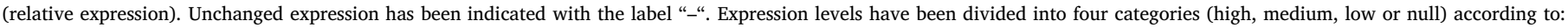

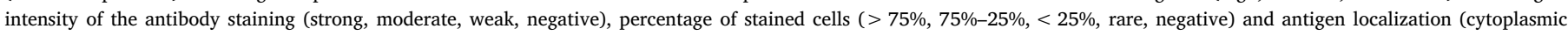
+ membranous + nuclear, cytoplasmic + membranous, nuclear, none)

proteins. The Drp1 inhibitor, mdivi-1, is so far the best-characterized drug that is able to affect mitochondrial dynamics [158]. It impairs Drp1 oligomerization on OMM and its ability to promote GTP hydrolysis [158]. Given the role of Drp1-mediated mitochondrial fission in tumor cell proliferation and metastatization, mdivi-1 has a potential anti-tumor role. Indeed, it can induce proliferation arrest of human breast and lung cancer cells $[22,67]$. Impaired assembly of mitotic spindle due to a hyperfused mitochondrial network after mdivi-1 treatment induces aneuploidy and consequent tumor cell apoptosis $[22,159]$. Despite the strong resistance to apoptosis often associated with an elongated mitochondrial network induced by mdivi-1 treatment particularly in cardiovascular cells and neurons [160], in brain tumor initiating cells (BTICs) (where Drp1 levels are upregulated), mdivi-1 treatment induces apoptosis, thus reducing BTICs tumor formation capacity [161]. Similarly, mdivi-1 increases the sensitivity of melanoma, lung cancer and osteosarcoma cells to Tumor Necrosis Factor-related apoptosis-inducer ligand (TRAIL)-induced caspase-dependent apoptosis [162], even though independently of its inhibitory action on Drp1 [163]. Mdivi-1 can cooperate with cisplatin to induce apoptosis in ovarian cancer cells in a Drp1-independent way [164], while it can have the opposite effect in invasive breast cancer cell lines [165]. Whether this pro-apoptotic role of mdivi-1 in regulating MOMP opening, and subsequent cytC release, is completely dependent on its inhibitory effect on Dpr1 or to its possible off-targets (such as MOMP assembly itself [166]) is currently not clarified. Indeed, in a few models, such as neuronal and MEF cell lines, mdivi-1 impinges on ROS levels and ETC complex I without affecting mitochondrial length or Drp1 [167]. Moreover, given the detrimental effect of prolonged alterations in mitochondrial morphology, the possibility to modulate locally and in a definite temporal window mitochondrial fission through mdivi-1 treatment could become a potential promising therapeutical anti-cancer treatment, although some concerns about its in vivo cytotoxicity must be previously resolved (see [160] for a review).

Besides mdivi-1, a recently discovered alternative Drp1 inhibitor, called p110, is able to block the Drp1-Fis1 interaction, thus preventing Drp1 recruitment to mitochondria, mitochondrial fragmentation and ROS production and consequently affecting apoptosis and cell viability. This has been observed for example in neuronal cell models of neurodegenerative diseases [168]. The potential role of this new inhibitor in the treatment of cancer is still unknown.

So far, M1 hydrazone is the only known drug capable to affect profusion mitochondrial shaping proteins [169]. Indeed, it is able to induce mitochondrial elongation and it strictly requires Opa-1 and at least Mfn1 or Mfn-2 presence [169]. Although shown to prevent cell death 


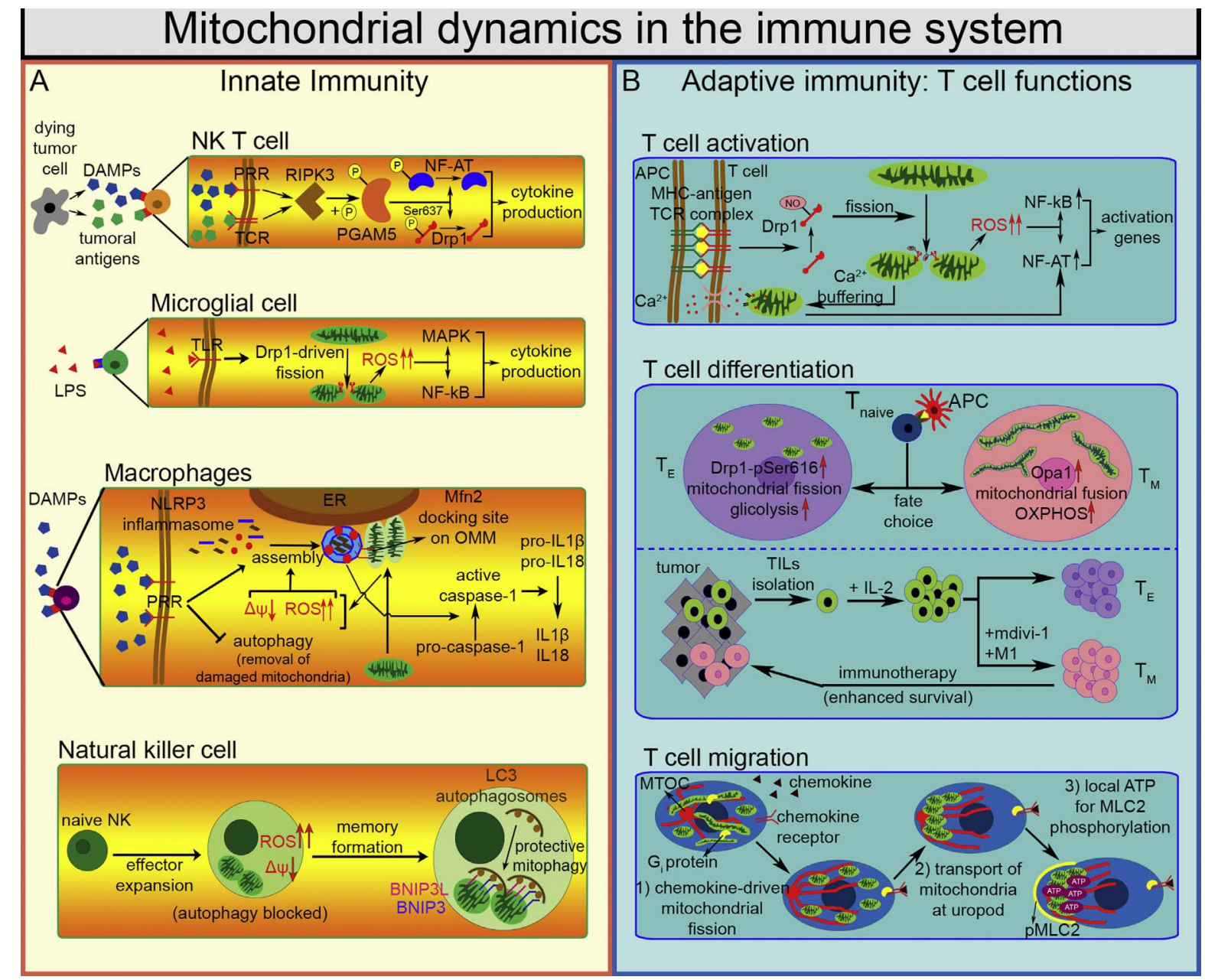

Fig. 2. Different roles played by the mitochondriashaping-proteins in the physiology of the immune system.

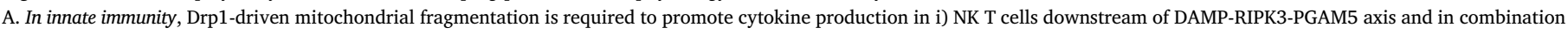

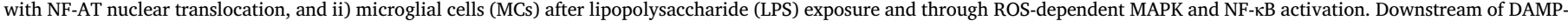

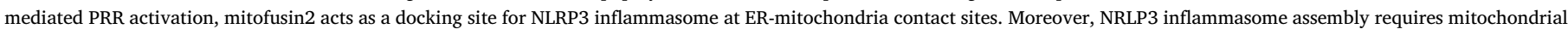

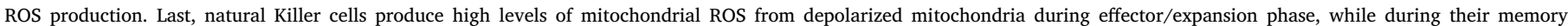
formation BNIP3/BNIP3L-mediated mitophagy removes damaged mitochondria. See text for details.

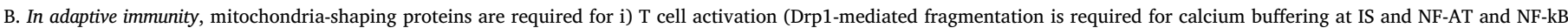

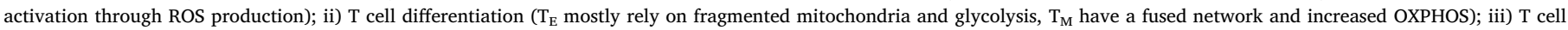

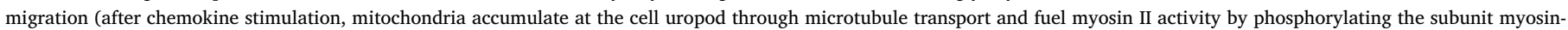

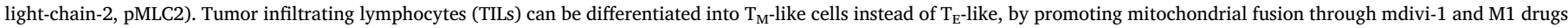

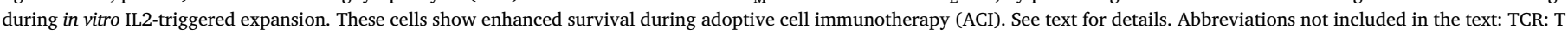

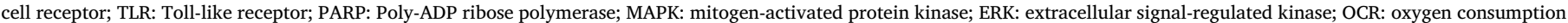
rate; SRC: spare respiratory capacity; M1: hydrazone M1.

associated with mitochondrial fragmentation [169], similarly to p110, its potential role as anti-cancer drug has still to be determined.

\section{Role of mitochondrial dynamics in the immune system and cancer immune-surveillance}

Although the weak immunogenicity of most tumor antigens, due to different factors spanning from low antigenicity to the immunomodulation of the TME (see [170] for a review), the activation of the immune response is our most important defence mechanism against cancer growth. Therefore, a complete understanding of the mitochondrial dynamics role in cancer growth cannot ignore the emerging evidence that connects them with different aspects of the immune system activity, in particular $\mathrm{T}$ cells. In recent years, new therapeutic approaches targeting the immune system have been studied and developed to inhibit tumor progression.

In the following paragraphs, we will provide an excursus on some of the effects that mitochondrial shape changes might differently have on immune cells and their activity, with particular attention to lymphocytes, in relation to cancer growth.

\subsection{Mitochondrial dynamics in innate immunity}

\subsubsection{Inflammation}

Stressed or dying tumor cells can release damage-associated molecular pattern (DAMP) molecules after cytotoxic treatments, such as ATP, HMGB1 and even DNA. Innate immune cells, such as dendritic cells (DCs), natural killer T (NKT) cells and macrophages, recognize DAMPs through pattern-recognition-receptors (PRRs) [171,172]. Once activated, these cells can directly kill the tumor cells in the TME or can migrate toward secondary lymphoid organs, where they activate cytotoxic CD8 + T cells of the adaptive immune response, which later migrate into the tumor mass recalled by local cytokines. Clearly, cancer cells can evade immunogenic cell death by acquiring mutations that affect the release of DAMP molecules or the response to apoptotic stimuli (see [173] for a review). Downstream of PRR and extracellular- 
ATP receptors activation, multimeric protein complexes, called inflammasomes, assemble in the cytosol and promote oligomerization of zymogen pro-caspase- 1 and its autoproteolytic cleavage in active caspase-1. This cleaves then pro-IL1 $\beta$ and pro-IL18 in their active pro-inflammatory forms [174]. Interestingly, mitochondria do play a role during the formation of inflammasome. NOD-like receptor containing pyrin domain 3 (NLRP3) inflammasome assembly requires mitochondrial ROS generation [175] and is associated with concurrent mitochondrial depolarization and mtDNA release [176]. It has also been suggested that autophagy/mitophagy inhibition could promote NLRP3 inflammasome activation by inducing mitochondrial dysfunction, which, in turn, increases again ROS production [175] and promoting cardiolipin accumulation on OMM, another signal triggering NLRP3 inflammasome assembly [177]. The active NLRP3 inflammasome clusters thus around ER and mitochondria [175]. That ROS accumulation is strictly required to activate inflammasome is demonstrated by the impairment in inflammasome activation upon antioxidant treatments such as (2R, 4R)-4-aminopyrrolidine-2-4-dicarboxylate (APDC) and $N$-acetyl-L-cysteine (NAC) [178]. Similarly, antioxidant mitoQ treatment reduces mitochondrial ROS amounts and reduces NLRP3 activation during inflammation [179]. NLRP3 deubiquitination is essential for inflammasome assembly and requires PRRs-derived ROS, while antioxidants can prevent also such regulation [180].

Together with ROS, also mitochondrial dynamics are implicated in inflammasome activation. Mitofusin-2 is able to interact directly with NLRP3, representing a docking site for inflammasome assembly on mitochondria [181]. Moreover, when PRRs bind viral antigens, they interact with the OMM-tethered mitochondrial adaptor mitochondrial antiviral signalling (MAVS) protein, which induces the activation of specific pro-inflammatory transcription factors, such as NF- $\mathrm{kB}$, which drives NLRP3 gene transcription [182]. MAVS interaction with Mfn-1 and -2 promotes mitochondrial elongation [183], which in turn favours MAVS association with ER-associated Stimulator of Interferon Genes (STING) protein, an interaction essential for the activation of downstream transcription factors [183]. The inflammasome requirement of mitochondrial elongation has been recently demonstrated also in Drp1silenced macrophages [184] and is correlated with reduced Opa-1 levels in rat [185]. Additionally, receptor-interacting protein kinase -1 and -3 (RIPK1/3)-dependent and lipopolysaccharide (LPS)-dependent inflammasome activation, and cytokine production upon viral infection, requires Drp1-dependent mitochondrial fission to induce mitochondrial damage and generate ROS [186,187]. The mitochondrial phosphatase phosphoglycerate mutase 5 (PGAM5) is the mediator of such RIPK-dependent Drp1 activation [188]. Similarly, microglial cells (MCs) in the central nervous system require Drp1-driven mitochondrial fragmentation and ROS signalling for correct cytokine production [189] (Fig. 2A).

\subsubsection{NK cell activity}

Natural killer (NK) cells are innate cytotoxic lymphocytes able to recognize host cells with abnormal MHC molecules expression and to induce their death through classical FasL or GranzymeB pathways. Thus, NK cells are able to recognize tumor cells, controlling in vivo tumor growth [190]. Recently, it has been discovered that NK cells may undergo memory formation with the generation of antigen-specific NK cells, even though - apparently, only during viral infection and not during cancer development [191]. While during their effector/expansion phase NK cells accumulates high amount of ROS, during the contraction-to-memory phase mitochondrial receptor BNIP3-BNIP3L proteins mediate removal of damaged mitochondria through mitophagy, thus reducing ROS amount and so promoting memory formation [192]. Although it has not been directly investigated whether or not mitochondrial dynamics are involved in this process, Drp1-dependent mitochondrial fission has the potential to play an important role in promoting mitochondrial clearance for the different reasons described above (Fig. 2A). Besides potentially regulating NK memory formation, mitochondrial dynamics are also possibly involved in the cytotoxic activity of these cells against cancer. Indeed, after stimulation, NK cells relocate and accumulate mitochondria at the NK-tumor cell contact-site (NK cell immune synapse) [193]. There, mitochondria control local calcium influx and supply elevated ATP amounts, thus sustaining exocytosis [193]. Due to the established role of the Drp1-dependent mitochondrial fission in controlling the organelles relocalization during the T cell immune synapse formation (see Section 3.2), it is plausible that the same process may act in NK cells, even though this still has to be proven. With this being the case, it would be then interesting to see whether mitochondrial dynamics might regulate also the release of cytotoxic granules by $\mathrm{T}$ lymphocytes.

In summary, although few works have, to date, specifically dissected the role of mitochondrial dynamics in modulating the innate immunity response during cancer growth, it is evident that they play an important role in activating inflammation and potentially in regulating NK activity during tumor growth. Therefore, these aspects are extremely promising, considering their crucial impact on the immunological response challenge and on cancer therapy.

\subsection{Mitochondrial dynamics in $T$ cell function}

Cytotoxic $\mathrm{CD}^{+}{ }^{+} \mathrm{T}$ lymphocytes (CTLs) represent the most specific line of defence against tumor growth in the organism. Once activated in secondary lymphoid organs, by tumor antigens presentation from specialised cells, $\mathrm{CD}^{+}$cells migrate into the tumor mass, looking for tumor cells to kill. Infiltrating CTLs are known as tumor infiltrating lymphocytes (TILs) and have been associated with better survival outcome in various melanomas and carcinomas [194,195]. Interestingly, TILs are becoming an interesting target for adoptive cell immunotherapy (ACI) [196].

\subsection{Cell activation}

An efficient $\mathrm{T}$ cell needs to be correctly activated through presentation of its specific antigen; it usually differentiates into different cell subtypes, and consequently has to proliferate in order to achieve the clonal expansion crucial for infection removal. An optimal T cell activation requires Drp1-dependent mitochondrial fragmentation to accumulate mitochondria beneath the TCR clusters on the plasma membrane, during the immune synapse (IS) formation [197]. In addition, Drp1-dependent mitochondria fragmentation is required at the IS for local calcium buffering and for the correct activation of several $\mathrm{T}$ cell transcription factors, such as NF-AT and NF- $\mathrm{kB}$, thus driving cytokine production [198-200] (Fig. 2C). Once activated, the subtype of effector $\mathrm{T}$ cells $\left(\mathrm{T}_{\mathrm{E}}\right)$ mainly rely on aerobic glycolysis to sustain their rapid proliferation rate [201]; this is achieved by fragmenting their mitochondrial network [202]. By contrast, memory $\mathrm{T}$ cells $\left(\mathrm{T}_{\mathrm{M}}\right)$ switch towards fatty-acid oxidation to sustain their metabolism and self-renewal, thus increasing their mitochondrial mass and sparing respiratory capacity (SRC) [201-204]. It has been recently shown that Opa-1 activity is essential to allow the correct formation and survival of $\mathrm{T}_{\mathrm{M}}$ cells, in which the mitochondrial network is clearly more elongated than in $\mathrm{T}_{\mathrm{E}}$ cells [202]. Pharmacological modulation of mitochondria-shaping proteins during in vitro $\mathrm{T}$ cell expansion, forcing mitochondria towards fusion, leads to the acquisition of a memory-like phenotype and to an increased survival rate, thus rendering $\mathrm{T}$ cells more effective in reducing tumor growth during adoptive cell immunotherapy (ACI) (Fig. 2B).

\subsection{Cell migration}

In line with what happens in fibroblast-like cell migration, highly polarised $\mathrm{T}$ cells need to accumulate their mitochondria during cell migration in a subcellular compartment, which -at variance with fibroblasts, is at the cell rear-edge (also known as uropod) [69,70]. Indeed, fragmentation of the organelles is essential to allow their 
accumulation at the cell uropod on a microtubules-dependent way. This is needed to locally produce high ATP amounts to fuel the migration process [69] (Fig. 2B). Consequently, shifting the mitochondria shape balance towards fusion impairs in vitro $\mathrm{T}$ cell polarization and migration. This is particularly interesting since in vivo tumor antigen-activated $\mathrm{T}$ cells need to extensively migrate to fully exploit their defensive functions. For example, cytotoxic $\mathrm{CD} 8{ }^{+} \mathrm{T}$ cell migration is required during at least four different steps for an efficient anti-tumor activity: i) extravasation of naïve $\mathrm{T}$ cells from the blood towards secondary lymphoid organs, where the tumor antigen can be met; ii) crawling migration inside lymph nodes to be correctly activated and differentiate; iii) extravasation across the blood barrier to join the tumor mass; iv) infiltration inside the tumor mass itself, to find and eliminate target cells. Interestingly, mitochondria accumulation at the $\mathrm{T}$ cell uropod during migration is required to locally promote phosphorylation and activation of the myosin-light-chain (MLC), a catalytic subunit of the myosin II [69]; indeed, it has been shown that myosin-deficient T cells do not correctly undergo extravasation and do not accumulate inside the tumor mass [205]. It would be interesting to further investigate the role of mitochondria-shaping proteins in the $\mathrm{T}$ cell function, from a motility viewpoint; of particular interest to us is what happens in the tumor immune-surveillance when mitochondria-shaping proteins are altered in T cell. Do the cells are still able to migrate and to infiltrate the tumor? By contrast, is the unbalance of mitochondrial dynamics much more detrimental for a $\mathrm{T}$ cell that needs to differentially extravasate and to correctly perform immune-surveillance? What does happen to the tumor growth if we would alternatively force mitochondria towards fusion or fission? Further studies in this direction would be extremely interesting.

\subsection{Cell exhaustion}

Emerging evidence in recent years show that TME can deeply affect the physiology of infiltrated $\mathrm{T}$ cells, thus favouring tumor immune escape. Tumor antigens are weakly immunogenic; $\mathrm{T}$ cells that enter TME are downregulated by a complex immunosuppressive environment consisting of cancer cells, inflammatory cells, stromal cells and immunosuppressive Tregs (see [206] for a review). Dysfunctional CD8 ${ }^{+}$ TILs are known as exhausted $\mathrm{T}$ cells $\left(\mathrm{T}_{\mathrm{EX}}\right)$. Accumulation of $\mathrm{T}_{\mathrm{EX}}$ cells in several tumors, (such as oesophageal cancer, HCC, soft tissue sarcoma, non-small lung cancer, breast cancer and melanoma) has been associated with poor survival outcome in patients (see [206] for a review). Additionally, TME can deeply affect the metabolic balance of $\mathrm{T}_{\mathrm{EX}}$ cells. Indeed, expression levels of the inhibitory receptors PD-1 and LAG-3 in TILs correlate with decreased mitochondrial mass and glucose uptake [207]. Given the importance of glucose availability to sustain $\mathrm{T}_{\mathrm{E}}$ glycolysis and effector functions [201], it becomes obvious that the glucose-limiting environment found in a tumor mass severely affects the anti-tumor immune response and cytokine production [208], thus favouring an imbalance towards $\mathrm{T}_{\mathrm{Ex}}$. TME can also lead to chronic Akt activation in TILs, which - in turn, downregulates the expression of PCG1 $\alpha$, the main promoter of mitochondrial biogenesis. Consequently, in these conditions, mitochondrial mass and function are severely impaired and cytokine production is reduced. Indeed, forcing PCG1 $\alpha$ expression in isolated TILs restores mitochondrial functionality and reduces tumor growth during ACI [207]. Considering the tight connection existing between mitochondrial metabolism and the shape of the mitochondrial network (see [209] for a review), we could expect an involvement of mitochondrial dynamics in TILs metabolism inside the tumor mass. Of note, it is still unknown whether or not differentiation to an exhausted phenotype in TILs could also affect mitochondrial dynamics, in addition to its well-established role on $\mathrm{T}_{\mathrm{Ex}}$ mitochondrial metabolism [207,210]. Recently, by a microarray analysis in chronic viral-infected patients fit has been found that exhausted CD8 $+\mathrm{T}$ cells show significant downregulation in the expression of several genes associated with mitochondrial OXPHOS metabolism, and upregulation of the oxidative stress pathway. Moreover, Opa-1 levels were found downregulated in that condition, this being probably linked to an altered respiration capacity, and MitoQ antioxidant treatment reduced ROS levels, increased mitochondrial membrane potential and restored cytokine production in $\mathrm{T}_{\mathrm{EX}}$ cells [211]. This suggests the existence of an interplay between mitochondrial dynamics, metabolism and ROS accumulation during $\mathrm{T}$ cell exhaustion that still needs to be investigated and might be crucial in cancer therapy. Indeed, this issue is from our viewpoint of particular interest, considering the possibility and beneficial potential of modulating mitochondrial dynamics in exhausted TILs alone or in combination with pharmacological treatments against $\mathrm{T}$ cell inhibitor receptors (such as anti-PD1 or anti-CTLA-4 antibodies, see [212] and [206] for reviews) to better restore $T_{E}$ cell phenotype during ACI.

\subsection{Cell apoptosis}

Recently, it has been demonstrated that in $\mathrm{T}$ cells, during Activation-Induced cell death (AICD: a physiological apoptotic process regulating the selection of functional thymocytes and the homeostasis of T lymphocytes in periphery), mitochondria undergo Drp1-dependent fragmentation, depolarization and cristae remodelling [213]. Depolarized mitochondria are targeted for mitophagy but, due to an early block of general autophagy, they are not removed, accumulate in the cell and amplify CytC release, thus priming cell death [213]. Tumor cells in the TME can increase FasL expression, thus promoting AICD in Fas Receptor-expressing cytotoxic T lymphocytes [214]. This tumor cell counterattack may limit the $\mathrm{T}$ cell anti-tumor immune response, thereby favouring cancer immune escape. Interestingly, AICD-resistant $\mathrm{T}$ cells may survive longer in the TME, showing enhanced effector function and inhibiting tumor growth [215]. The fact that AICD is at least in part regulated by the mitochondrial dynamics-autophagy cross talk, suggest that their manipulation to increase apoptosis resistance in TILs may represent an interesting therapeutic target to enhance an antitumor immune response.

\subsection{Targeting mitochondrial dynamics to improve the anti-cancer immune response}

Mitochondrial dynamics have been shown, in recent years, to be involved in controlling several functions of both adaptive and innate immune response. In sum, remodelling of the mitochondrial network is required for establishing local inflammation through inflammasome activation, for controlling NK cytotoxicity and for activation, migration, differentiation and survival of $\mathrm{T}$ lymphocytes. The possibility to isolate immune cells from cancer patients and to cultivate them in vitro would also be a crucial technical achievement to consequently re-shape the mitochondrial network before using these cells in ACI treatments. This could be done also in combination with current therapeutic strategies to improve these cells anti-cancer efficacy. Although no attempts in this direction have been made so far, we may hope to manipulate in the future mitochondrial morphology of isolated and tumor-specific immune cells to confer them higher effector functions, more invasiveness capability or stronger survival in the TME, once inserted back into the patient. However, a full comprehension of the roles played by mitochondrial dynamics in different phases of leukocyte activation is strongly required before proceeding, eventually, with a therapeutic step.

Inflammation is emerging as a potent driver of tumor progression. While during initial cancer onset the activation of inflammation by the immune system can recruit cytotoxic lymphocytes in the TME, thus mediating tumor immune-destruction, chronic inflammation may favour at later stages the development of an immune-suppressive environment, further promoting tumor growth [216]. For example, ROS species generated during lymphocyte activity may increase DNA mutational load in cancer cells and tumor-associated macrophages (TAMs) 
can produce angiogenic factors, thus recruiting new capillaries inside the tumor mass. TAMs also release IL-10, a cytokine that inhibits $\mathrm{T}$ cell cytotoxicity [217]. The possibility of modulating the inflammatory response in the TME by influencing the mitochondrial morphology of innate immune system cells is a possibility still not explored. However, in vivo pharmacological treatments, such as those using mdivi-1, to decrease tumor cells proliferation and apoptosis-resistance may promote, in the meanwhile, further inflammasome activation in the TME, thus favouring tumor survival from another angle. This highlights the difficulties for each single treatment to fight efficiently against cancer growth, given the complex and multiple environment found inside the tumor mass.

As regards the anti-tumor $\mathrm{T}$ cell response, forcing mitochondrial fusion during in vitro $\mathrm{T}$ cell expansion promotes differentiation of naïve T cells toward a memory phenotype, as recently demonstrated [202]. This can confer a higher survival to these cells during ACI. However, T cell migration strictly depends on optimal fragmentation of the mitochondrial network [69]. This suggests that, once back in the patient, $\mathrm{T}$ cells may infiltrate better inside the TME if the mitochondrial network is genetically reprogrammed towards a more fragmented state, for example by chronically altering the expression of mitochondria-shaping proteins. Fragmentation of the network may favour cytotoxicity of infiltrating T cells, similarly to what has been reported for NK cells [193], even though also this aspect remains a speculation. Nevertheless, constitutive fragmentation of the mitochondrial network may increase TILs susceptibility to AICD [213]. Therefore, although more cells might accumulate in the TME in that context, they could also die faster. In addition, since mitochondrial fragmentation is frequently associated with a glycolytic metabolism, TILs with fragmented mitochondria may suffer from low availability of glucose in the TME. For this reason, reprogramming TILs mitochondria towards a fused state may increase OXPHOS metabolism, so favouring their survival. Last, nothing is known yet about the modifications that the mitochondrial morphology undergo during $\mathrm{T}$ cell exhaustion and whether its modulation might somehow prevent or restore $\mathrm{T}$ cell functionality. For example, exhausted T cells show motility paralysis during viral infection [218] and this depends on downregulation of the MAPK/Erk pathway [218], which is known to positively regulate Drp1 activation [147]. Since Drp1 overexpression promotes T cell migration [69], could Drp1-dependent fragmentation play a role in $\mathrm{T}_{\mathrm{EX}}$ migration? Would it be feasible to increase $\mathrm{T}_{\mathrm{EX}}$ cell motility by altering mitochondrial morphology? In addition, could mitochondrial dynamics represent a potential target to reprogram $\mathrm{T}$ cell exhaustion metabolism? All these questions are still interestingly open and unsolved. Given the plasticity of the mitochondrial network remodelling, $\mathrm{T}$ cells may adapt the morphology of the organelle according to their needs, once in the TME. This suggests that a one-way modulation of mitochondria-shaping proteins activity could hardly result in successful ACI, and that developing new strategies for fine-tuning $\mathrm{T}$ cell mitochondria morphology in the TME should be of the highest priority.

\section{Conclusions}

Mitochondrial dynamics and the related mitochondria-shaping proteins tightly regulate several processes required for tumor onset, growth and metastatization. Their alterations are very common in different human cancers and could be used as predictive tools to define specific chemotherapeutic approaches. Indeed, in this new era of personalized medicine, specific pharmacological treatments modulating mitochondrial dynamics are increasingly adopted during cancer therapy and the development of new strategies able to specifically impact on the mitochondrial shape is a high priority in cancer therapy. Various cancers (and even diverse stages of the same cancer) may profit from different states of the mitochondrial network, particularly regarding the response to apoptosis-inducing drugs. Based on data here reported, it is quite evident that no single therapy affecting mitochondrial dynamics can be successful in all cancer treatments, but the identification of specific mitochondrial signatures in patients with different cancers is required in order to adopt the best strategy. The picture is even more complex, given the roles that mitochondrial dynamics play in the innate and adaptive immune system functions. Although this area is still new, the possibility to modulate in vitro mitochondrial dynamics on TILs offers alternative strategies to control tumor growth by helping the immune system to fight against it. On the other hand, it is important to bear in mind that pharmacological treatment to modulate mitochondrial dynamics in tumor cells may indirectly affect the immune response, or vice versa. Therefore, the development of new strategies that can efficiently hit the tumor twice, acting both on tumor cells and on immune-surveillance, is becoming even more urgent.

\section{Conflict of interest}

Authors declare no conflict of interest.

\section{Funding}

This work was funded by the Italian Ministry of Health (GR-201102351643) to SC and by grants from the Italian AIRC (IG2016-18906) and Fondazione Roma.

\section{References}

[1] S.A. Detmer, D.C. Chan, Functions and dysfunctions of mitochondrial dynamics, Nature Reviews Molecular Cell Biology, Nature Publishing Group, 2007, pp. 870-879.

[2] A. Santel, S. Frank, B. Gaume, M. Herrler, R.J. Youle, M.T. Fuller, Mitofusin-1 protein is a generally expressed mediator of mitochondrial fusion in mammalian cells, J. Cell Sci. 116 (2003) 2763-2774.

[3] Y. Eura, N. Ishihara, S. Yokota, K. Mihara, Two mitofusin proteins, mammalian homologues of FZO, with distinct functions are both required for mitochondrial fusion, J. Biochem. 134 (2003) 333-344.

[4] Y. Qi, L. Yan, C. Yu, X. Guo, X. Zhou, X. Hu, et al., Structures of human mitofusin 1 provide insight into mitochondrial tethering, J. Cell Biol. 215 (2016) 621-629.

[5] S. Pich, D. Bach, P. Briones, M. Liesa, M. Camps, X. Testar, et al., The CharcotMarie-Tooth type 2A gene product, Mfn2, up-regulates fuel oxidation through expression of OXPHOS system, Hum. Mol. Genet. 14 (2005) 1405-1415.

[6] E.S. Ramos, N.-G.r. Larsson, A. Mourier, Bioenergetic roles of mitochondrial fusion, Biochim. Biophys. Acta (BBA)-Bioenerg. 1857 (2016) 1277-1283.

[7] O.M. de Brito, L. Scorrano, Mitofusin 2 tethers endoplasmic reticulum to mitochondria, Nature 456 (7222) (2008) 605-610.

[8] S. Cipolat, O.M. de Brito, B. Dal Zilio, L. Scorrano, OPA1 requires mitofusin 1 to promote mitochondrial fusion, Proc. Natl. Acad. Sci. U. S. A. 101 (2004) $15927-15932$

[9] R. Anand, T. Wai, M.J. Baker, N. Kladt, A.C. Schauss, E. Rugarli, et al., The i-AAA protease YME1L and OMA1 cleave OPA1 to balance mitochondrial fusion and fission, J. Cell Biol. 204 (2014) 919-929.

[10] P. Paumard, J. Vaillier, B. Coulary, J. Schaeffer, V. Soubannier, D.M. Mueller, et al., The ATP synthase is involved in generating mitochondrial cristae morphology, EMBO J. 21 (2002) 221-230.

[11] V. Kozjak-Pavlovic, The MICOS complex of human mitochondria, Cell Tissue Res. 367 (2017) 83-93.

[12] C. Frezza, S. Cipolat, O.M. de Brito, M. Micaroni, G.V. Beznoussenko, T. Rudka, et al., OPA1 controls apoptotic cristae remodeling independently from mitochondrial fusion, Cell 126 (2006) 177-189.

[13] T. Varanita, M.E. Soriano, V. Romanello, T. Zaglia, R.n. Quintana-Cabrera, M. Semenzato, et al., The OPA1-dependent mitochondrial cristae remodeling pathway controls atrophic, apoptotic, and ischemic tissue damage, Cell Metab. 21 (2015) 834-844.

[14] M.V. Alavi, N. Fuhrmann, Dominant optic atrophy OPA1, and mitochondrial quality control: understanding mitochondrial network dynamics, Mol. Neurodegener. 8 (1) (2013) p1.

[15] H. Chen, M. Vermulst, Y.E. Wang, A. Chomyn, T.A. Prolla, J.M. McCaffery, et al., Mitochondrial fusion is required for mtDNA stability in skeletal muscle and tolerance of mtDNA mutations, Cell 141 (2010) 280-289.

[16] S. Feely, M. Laura, C. Siskind, S. Sottile, M. Davis, V. Gibbons, et al., MFN2 mutations cause severe phenotypes in most patients with CMT2A, Neurology 76 (2011) 1690-1696.

[17] M. Ranieri, R. Del Bo, A. Bordoni, D. Ronchi, I. Colombo, G. Riboldi, et al., Optic atrophy plus phenotype due to mutations in the OPA1 gene: two more Italian families, J. Neurol. Sci. 315 (2012) 146-149.

[18] A. Gal, P. Balicza, D. Weaver, S. Naghdi, S.K. Joseph, P. Várnai, et al., MSTO1 is a cytoplasmic pro-mitochondrial fusion protein, whose mutation induces myopathy 
and ataxia in humans, EMBO Mol. Med. (2017) e201607058.

[19] G. Twig, O.S. Shirihai, The interplay between mitochondrial dynamics and mitophagy, Antioxid. Redox Signal. 14 (10) (2011) 1939-1951.

[20] H.-F. Jheng, P.-J. Tsai, S.-M. Guo, L.-H. Kuo, C.-S. Chang, I.-J. Su, et al., Mitochondrial fission contributes to mitochondrial dysfunction and insulin resistance in skeletal muscle, Mol. Cell. Biol. 32 (2012) 309-319.

[21] K. Mitra, Mitochondrial fission-fusion as an emerging key regulator of cell proliferation and differentiation, Bioessays 35 (11) (2013) 955-964.

[22] W. Qian, J. Wang, V. Roginskaya, L.A. McDermott, R.P. Edwards, D.B. Stolz, et al., Novel combination of mitochondrial division inhibitor 1 (mdivi-1) and platinum agents produces synergistic pro-apoptotic effect in drug resistant tumor cells, Oncotarget 5 (2014) 4180.

[23] N. Taguchi, N. Ishihara, A. Jofuku, T. Oka, K. Mihara, Mitotic phosphorylation of dynamin-related GTPase Drp1 participates in mitochondrial fission, J. Biol. Chem. 282 (2007) 11521-11529.

[24] R.J. Youle, M. Karbowski, Mitochondrial fission in apoptosis, Nat. Rev. Mol. Cell Biol. 6 (8) (2005) 657-663.

[25] S. Wasiak, R. Zunino, H.M. McBride, Bax/Bak promote sumoylation of DRP1 and its stable association with mitochondria during apoptotic cell death, J. Cell Biol. 177 (3) (2007) 439-450.

[26] H. Otera, N. Ishihara, K. Mihara, New insights into the function and regulation of mitochondrial fission, Biochim. Biophys. Acta (BBA)-Mol. Cell Res. 1833 (5) (2013) 1256-1268

[27] J.R. Friedman, L.L. Lackner, M. West, J.R. DiBenedetto, J. Nunnari, G.K. Voeltz ER tubules mark sites of mitochondrial division, Science 334 (2011) 358-362.

[28] S. Li, S. Xu, B.A. Roelofs, L. Boyman, W.J. Lederer, H. Sesaki, et al., Transient assembly of F-actin on the outer mitochondrial membrane contributes to mitochondrial fission, J. Cell Biol. 208 (2015) 109-123.

[29] J.M. Shaw, J. Nunnari, Mitochondrial dynamics and division in budding yeast, Trends Cell Biol. 12 (4) (2002) 178-184.

[30] E. Smirnova, L. Griparic, D.-L. Shurland, A.M. Van Der Bliek, Dynamin-related protein Drp1 is required for mitochondrial division in mammalian cells, Mol. Biol. Cell 12 (2001) 2245-2256.

[31] E. Ingerman, E.M. Perkins, M. Marino, J.A. Mears, J.M. McCaffery, J.E. Hinshaw, et al., Dnm1 forms spirals that are structurally tailored to fit mitochondria, J. Cell Biol. 170 (2005) 1021-1027.

[32] N. Ishihara, M. Nomura, A. Jofuku, H. Kato, S.O. Suzuki, K. Masuda, et al., Mitochondrial fission factor Drp1 is essential for embryonic development and synapse formation in mice, Nat. Cell Biol. 11 (2009) 958-966.

[33] D.I. James, P.A. Parone, Y. Mattenberger, J.-C. Martinou, hFis1, a novel component of the mammalian mitochondrial fission machinery, J. Biol. Chem. 278 (2003) 36373-36379.

[34] H. Otera, K. Mihara, Discovery of the membrane receptor for mitochondrial fission GTPase Drp1, Small GTPases 3 (2011) 167-172.

[35] H. Otera, C. Wang, M.M. Cleland, K. Setoguchi, S. Yokota, R.J. Youle, et al., Mff is an essential factor for mitochondrial recruitment of Drp1 during mitochondrial fission in mammalian cells, J. Cell Biol. 191 (2010) 1141-1158.

[36] S. Gandre-Babbe, A.M. van der Bliek, The novel tail-anchored membrane protein Mff controls mitochondrial and peroxisomal fission in mammalian cells, Mol. Biol. Cell 19 (6) (2008) 2402-2412.

[37] C.S. Palmer, L.D. Osellame, D. Laine, O.S. Koutsopoulos, A.E. Frazier, M.T. Ryan, MiD49 and MiD51, new components of the mitochondrial fission machinery, EMBO Rep. 12 (2011) 565-573.

[38] X. Wang, M.H. Yan, H. Fujioka, J. Liu, A. Wilson-Delfosse, S.G. Chen, et al., LRRK2 regulates mitochondrial dynamics and function through direct interaction with DLP1, Hum. Mol. Genet. 21 (2012) 1931-1944.

[39] J. Niu, M. Yu, C. Wang, Z. Xu, Leucine-rich repeat kinase 2 disturbs mitochondrial dynamics via Dynamin-like protein, J. Neurochem. 122 (2012) 650-658.

[40] N.M. Alto, J. Soderling, J.D. Scott, Rab32 is an A-kinase anchoring protein and participates in mitochondrial dynamics, J. Cell Biol. 158 (4) (2002) 659-668.

[41] H. Harada, B. Becknell, M. Wilm, M. Mann, L.J.-s. Huang, S.S. Taylor, et al., Phosphorylation and inactivation of BAD by mitochondria-anchored protein kinase A, Mol. Cell 3 (1999) 413-422.

[42] J.E. Lee, L.M. Westrate, H. Wu, C. Page, G.K. Voeltz, Multiple dynamin family members collaborate to drive mitochondrial division, Nature 540 (2016) 139-143.

[43] H. Li, Y. Ruan, K. Zhang, F. Jian, C. Hu, L. Miao, et al., Mic60/Mitofilin determines MICOS assembly essential for mitochondrial dynamics and mtDNA nucleoid organization, Cell Death Differ. 23 (2016) 380-392.

[44] C. Ott, E. Dorsch, M. Fraunholz, S. Straub, V. Kozjak-Pavlovic, Detailed analysis of the human mitochondrial contact site complex indicate a hierarchy of subunits, PLoS One 10 (2015) e0120213.

[45] D. Hanahan, R.A. Weinberg, Hallmarks of cancer: the next generation, Cell 144 (2011) 646-674

[46] M. Karbowski, Y.-J. Lee, B. Gaume, S.-Y. Jeong, S. Frank, A. Nechushtan, et al., Spatial and temporal association of Bax with mitochondrial fission sites, Drp1, and Mfn2 during apoptosis, J. Cell Biol. 159 (2002) 931-938.

[47] P. Wang, P. Wang, B. Liu, J. Zhao, Q. Pang, S.G. Agrawal, et al., Dynamin-related protein Drp1 is required for Bax translocation to mitochondria in response to irradiation-induced apoptosis, Oncotarget 6 (2015) 22598.

[48] S. Montessuit, S.P. Somasekharan, O. Terrones, S. Lucken-Ardjomande, S.b. Herzig, R. Schwarzenbacher, et al., Membrane remodeling induced by the dynamin-related protein Drp1 stimulates Bax oligomerization, Cell 142 (2010) 889-901.

[49] M. Karbowski, K.L. Norris, M.M. Cleland, S.-Y. Jeong, R.J. Youle, Role of Bax and Bak in mitochondrial morphogenesis, Nature 443 (2006) 658-662.

[50] H. Li, Y. Chen, A.F. Jones, R.H. Sanger, L.P. Collis, R. Flannery, et al., Bcl-xL induces Drp1-dependent synapse formation in cultured hippocampal neurons, Proc. Natl. Acad. Sci. U. S. A. 105 (2008) 2169-2174.

[51] T.T. Renault, K.V. Floros, R. Elkholi, K.-A. Corrigan, Y. Kushnareva, S.Y. Wieder, et al., Mitochondrial shape governs BAX-induced membrane permeabilization and apoptosis, Mol. Cell 57 (2015) 69-82.

[52] R. Yamaguchi, L. Lartigue, G. Perkins, R.T. Scott, A. Dixit, Y. Kushnareva, et al., Opa1-mediated cristae opening is Bax/Bak and BH3 dependent, required for apoptosis, and independent of Bak oligomerization, Mol. Cell 31 (2008) 557-569.

[53] C. Merkwirth, S. Dargazanli, T. Tatsuta, S. Geimer, B. Lower, F.T. Wunderlich, et al., Prohibitins control cell proliferation and apoptosis by regulating OPA1dependent cristae morphogenesis in mitochondria, Genes. Dev. 22 (2008) 476-488.

[54] A. Pyakurel, C. Savoia, D. Hess, L. Scorrano, Extracellular regulated kinase phosphorylates mitofusin 1 to control mitochondrial morphology and apoptosis, Mol. Cell 58 (2015) 244-254.

[55] A. Inoue-Yamauchi, H. Oda, Depletion of mitochondrial fission factor DRP1 causes increased apoptosis in human colon cancer cells, Biochem. Biophys. Res. Commun. 421 (1) (2012) 81-85.

[56] J. Chiche, M. Rouleau, P. Gounon, M.C. Brahimi-Horn, J. Pouysségur, N.M. Mazure, Hypoxic enlarged mitochondria protect cancer cells from apoptotic stimuli, J. Cell. Physiol. 222 (2010) 648-657.

[57] Y.-Y. Chiang, S.-L. Chen, Y.-T. Hsiao, C.-H. Huang, T.-Y. Lin, I.-P. Chiang, et al., Nuclear expression of dynamin-related protein 1 in lung adenocarcinomas, Mod. Pathol. 22 (2009) 1139-1150.

[58] S. Fan, B. Liu, L. Sun, X.-b. Lv, Z. Lin, W. Chen, et al., Mitochondrial fission determines cisplatin sensitivity in tongue squamous cell carcinoma through the BRCA1-miR-593-5p-MFF axis, Oncotarget 6 (2015) 14885.

[59] G. Santin, V.M. Piccolini, S. Barni, P. Veneroni, V. Giansanti, V. Dal Bo, et al. Mitochondrial fusion: a mechanism of cisplatin-induced resistance in neuroblastoma cells? Neurotoxicology 34 (2013) 51-60.

[60] L. Benetatos, E. Hatzimichael, E. Londin, G. Vartholomatos, P. Loher, I. Rigoutsos, et al., The microRNAs within the DLK1-DIO3 genomic region: involvement in disease pathogenesis, Cell. Mol. Life Sci. 70 (2013) 795-814.

[61] P. Dutta, E. Haller, A. Sharp, M. Nanjundan, MIR494 reduces renal cancer cell survival coinciding with increased lipid droplets and mitochondrial changes, BMC Cancer 16 (2016) 33.

[62] B. Magi, A. Ettorre, S. Liberatori, L. Bini, M. Andreassi, S. Frosali, et al., Selectivity of protein carbonylation in the apoptotic response to oxidative stress associated with photodynamic therapy: a cell biochemical and proteomic investigation, Cell Death Differ. 11 (2004) 842-852.

[63] J. An, J. Shi, Q. He, K. Lui, Y. Liu, Y. Huang, et al., CHCM1/CHCHD6, novel mitochondrial protein linked to regulation of mitofilin and mitochondrial cristae morphology, J. Biol. Chem. 287 (2012) 7411-7426.

[64] D. Faccenda, J. Nakamura, G. Gorini, G.K. Dhoot, M. Piacentini, M. Yoshida, et al., Control of mitochondrial remodeling by the ATPase inhibitory factor 1 unveils a pro-survival relay via OPA1, Cell Rep. 18 (2017) 1869-1883.

[65] D.K. Tanwar, D.J. Parker, P. Gupta, B. Spurlock, R.D. Alvarez, M.K. Basu, et al., Crosstalk between the mitochondrial fission protein, Drp1, and the cell cycle is identified across various cancer types and can impact survival of epithelial ovarian cancer patients, Oncotarget 7 (37) (2016) 60021-60037.

[66] L. Zhan, H. Cao, G. Wang, Y. Lyu, X. Sun, J. An, et al., Drp1-mediated mitochondrial fission promotes cell proliferation through crosstalk of p53 and NF-kB pathways in hepatocellular carcinoma, Oncotarget 7 (40) (2016) 65001-65011.

[67] J. Rehman, H.J. Zhang, P.T. Toth, Y. Zhang, G. Marsboom, Z. Hong, et al., Inhibition of mitochondrial fission prevents cell cycle progression in lung cancer, FASEB J. 26 (2012) 2175-2186.

[68] C. Sievers, G. Billig, K. Gottschalk, T. Rudel, Prohibitins are required for cancer cell proliferation and adhesion, PLoS One 5 (2010) e12735.

[69] S. Campello, R.A. Lacalle, M. Bettella, S. Manes, L. Scorrano, A. Viola, Orchestration of lymphocyte chemotaxis by mitochondrial dynamics, J. Exp. Med. 203 (2006) 2879-2886.

[70] A.F. Da Silva, C. Valacca, E. Rios, H. Pòpulo, P. Soares, M. Sobrinho-Simones, et al., Mitochondrial dynamics protein Drp1 is overexpressed in oncocytic thyroid tumors and regulates cancer cell migration, PLoS One 10 (2015) e0122308.

[71] J. Zhao, J. Zhang, M. Yu, Y. Xie, Y. Huang, D.W. Wolff, et al., Mitochondrial dy namics regulates migration and invasion of breast cancer cells, Oncogene 32 (2013) 4814-4824.

[72] T.-F. Che, C.-W. Lin, Y.-Y. Wu, Y.-J. Chen, C.-L. Han, Y.-1. Chang, et al., Mitochondrial translocation of EGFR regulates mitochondria dynamics and promotes metastasis in NSCLC, Oncotarget 6 (2015) 37349.

[73] S.P. Desai, S.N. Bhatia, M. Toner, D. Irimia, Mitochondrial localization and the persistent migration of epithelial cancer cells, Biophys. J. 104 (2013) 2077-2088.

[74] Y.-Y. Wan, J.-F. Zhang, Z.-J. Yang, L.-P. Cassidy-Stone ng, Y.F. Wei, Q.-N. Lai, et al., Involvement of Drp1 in hypoxia-induced migration of human glioblastoma U251 cells, Oncol. Rep. 32 (2014) 619-626.

[75] X.-J. Han, Z.-J. Yang, L.-P. Jiang, Y.-F. Wei, M.-F. Liao, Y. Qian, et al., Mitochondrial dynamics regulates hypoxia-induced migration and antineoplastic activity of cisplatin in breast cancer cells, Int. J. Oncol. 46 (2015) 691-700.

[76] M.C. Caino, J.H. Seo, A. Aguinaldo, E. Wait, K.G. Bryant, A.V. Kossenkov, et al., A neuronal network of mitochondrial dynamics regulates metastasis, Nat. Commun. 7 (2016).

[77] J.-U. Jung, S. Ravi, D.W. Lee, K. McFadden, M.L. Kamradt, L.G. Toussaint, et al. NIK/MAP3K14 regulates mitochondrial dynamics and trafficking to promote cell invasion, Curr. Biol. 26 (2016) 3288-3302.

[78] L. Wang, Y. Yoon, Mitochondrial fission is necessary for vascular smooth muscle cell migration (761.1), FASEB J. 28 (2014). 
[79] M.C. Caino, J.C. Ghosh, Y.C. Chae, V. Vaira, D.B. Rivadeneira, A. Faversani, et al., PI3K therapy reprograms mitochondrial trafficking to fuel tumor cell invasion, Proc. Natl. Acad. Sci. U. S. A. 112 (2015) 8638-8643.

[80] R. Rossignol, R. Gilkerson, R. Aggeler, K. Yamagata, S.J. Remington, R.A. Capaldi, Energy substrate modulates mitochondrial structure and oxidative capacity in cancer cells, Cancer Res. 64 (2004) 985-993.

[81] H. Chen, A. Chomyn, D.C. Chan, Disruption of fusion results in mitochondria heterogeneity and dysfunction, J. Biol. Chem. 280 (28) (2005) 26185-26192.

[82] D. Loiseau, A. Chevrollier, C. Verny, V. Guillet, N.g. Gueguen, M.A. Pou De Crescenzo, et al., Mitochondrial coupling defect in Charcot-Marie-Tooth type 2A disease, Ann. Neurol. 61 (2007) 315-323.

[83] M. Picard, O.S. Shirihai, B.J. Gentil, Y. Burelle, Mitochondrial morphology transitions and functions: implications for retrograde signaling? Am. J. Physiol. Regul. Integr. Comp. Physiol. 304 (2013) R393-R406.

[84] D.A. Patten, J. Wong, M. Khacho, V. Soubannier, R.J. Mailloux, K. Pilon-Larose, et al., OPA1-dependent cristae modulation is essential for cellular adaptation to metabolic demand, EMBO J. (2014) e201488349.

[85] S. Pich, D. Bach, P. Briones, M. Liesa, M. Camps, X. Testar, et al., The CharcotMarie-Tooth type 2A gene product, Mfn2, up-regulates fuel oxidation through expression of OXPHOS system, Hum. Mol. Genet. 14 (2005) 1405-1415.

[86] P.A. Parone, S. Da Cruz, D. Tondera, Y. Mattenberger, D.I. James, P. Maechler, et al., Preventing mitochondrial fission impairs mitochondrial function and leads to loss of mitochondrial DNA, PLoS One 3 (2008) e3257.

[87] J. Prieto, M. Leòn, X. Ponsoda, R.n. Sendra, R. Bort, R. Ferrer-Lorente, et al., Early ERK1/2 activation promotes DRP1-dependent mitochondrial fission necessary for cell reprogramming, Nat. Commun. 7 (2016).

[88] A.S. Rambold, B. Kostelecky, N. Elia, J. Lippincott-Schwartz, Tubular network formation protects mitochondria from autophagosomal degradation during nutrient starvation, Proc. Natl. Acad. Sci. U. S. A. 108 (2011) 10190-10195.

[89] J.-Y. Lee, M. Kapur, M. Li, M.-C. Choi, S. Choi, H.-J. Kim, et al., MFN1 deacetylation activates adaptive mitochondrial fusion and protects metabolically challenged mitochondria, J. Cell Sci. 127 (2014) 4954-4963.

[90] M.O. Dietrich, Z.-W. Liu, T.L. Horvath, Mitochondrial dynamics controlled by mitofusins regulate Agrp neuronal activity and diet-induced obesity, Cell 155 (2013) 188-199.

[91] O. Warburg, On the origin of cancer cells, Science 123 (3191) (1956) 309-314.

[92] D. Wallace, Mitochondria and cancer: Warburg addressed, Cold Spring Harbor Symposia on Quantitative Biology, Cold Spring Harbor Laboratory Press, 2005.

[93] F. Maddalena, G. Lettini, R. Gallicchio, L. Sisinni, V. Simeon, A. Nardelli, et al., Evaluation of glucose uptake in normal and cancer cell lines by positron emission tomography, Mol. Imaging 14 (2015) 7290.2015.00021.

[94] M.I. Koukourakis, A. Giatromanolaki, A.L. Harris, E. Sivridis, Comparison of metabolic pathways between cancer cells and stromal cells in colorectal carcinomas: a metabolic survival role for tumor-associated stroma, Cancer Res. 66 (2006) 632-637.

[95] M. Sciacovelli, E. Gonzalves, T.I. Johnson, V.R. Zecchini, A.S.H. da Costa, E. Gaude, et al., Fumarate is an epigenetic modifier that elicits epithelial-to-mesenchymal transition, Nature 537 (2016) 544-547.

[96] M. Sciacovelli, C. Frezza, Fumarate drives EMT in renal cancer, Cell Death Differ. 24 (1) (2016) 1-2.

[97] E. Hervouet, J. Demont, P. Pecina, A. Vojtìskova, J. Houstek, H.l.n. Simonnet, et al., A new role for the von Hippel-Lindau tumor suppressor protein: stimulation of mitochondrial oxidative phosphorylation complex biogenesis, Carcinogenesis 26 (2005) 531-539.

[98] T.J. Schulz, R. Thierbach, A. Voigt, G. Drewes, B. Mietzner, P. Steinberg, et al., Induction of oxidative metabolism by mitochondrial frataxin inhibits cancer growth Otto Warburg Revisited, J. Biol. Chem. 281 (2006) 977-981.

[99] L. Plecità-Hlavatà, M. Lessard, J. Santorovà, J. Bewersdorf, P. Jezek, Mitochondrial oxidative phosphorylation and energetic status are reflected by morphology of mitochondrial network in INS-1E and HEP-G2 cells viewed by 4Pi microscopy, Biochim. Biophys. Acta (BBA)—Bioenerg. 1777 (2008) 834-846.

[100] J. Hagenbuchner, A. Kuznetsov, P. Obexer, M. Ausserlechner, BIRC5/Survivin enhances aerobic glycolysis and drug resistance by altered regulation of the mitochondrial fusion/fission machinery, Oncogene 32 (2013) 4748-4757.

[101] E.G. Konstantakou, G.E. Voutsinas, A.D. Velentzas, A.-S. Basogianni, E. Paronis, E. Balafas, et al., 3-BrPA eliminates human bladder cancer cells with highly on cogenic signatures via engagement of specific death programs and perturbation of multiple signaling and metabolic determinants, Mol. Cancer 14 (2015) 135.

[102] U. Dier, D.-H. Shin, L.M.P. Hemachandra, L.M. Uusitalo, N. Hempel, Bioenergetic analysis of ovarian cancer cell lines: profiling of histological subtypes and identification of a mitochondria-defective cell line, PLoS One 9 (2014) e98479.

[103] M. Van Gisbergen, A. Voets, M. Starmans, I. de Coo, R. Yadak, R. Hoffmann, et al., How do changes in the mtDNA and mitochondrial dysfunction influence cancer and cancer therapy? Challenges, opportunities and models, Mutat. Res. 764 (2015) 16-30.

[104] E. Reznik, M.L. Miller, Y. Senbabaoglu, N. Riaz, J. Sarungbam, S.K. Tickoo, et al., Mitochondrial DNA copy number variation across human cancers, eLife 5 (2016) e10769.

[105] P.A. Parone, S. Da Cruz, D. Tondera, Y. Mattenberger, D.I. James, P. Maechler, et al., Preventing mitochondrial fission impairs mitochondrial function and leads to loss of mitochondrial DNA, PLoS One 3 (2008) e3257.

[106] M. Schieber, N.S. Chandel, ROS function in redox signaling and oxidative stress, Curr. Biol. 24 (2014) R453-R462.

[107] E. Birben, U.M. Sahiner, C. Sackesen, S. Erzurum, O. Kalayci, Oxidative stress and antioxidant defense, World Allergy Organ. J. 5 (2012) 9.

[108] S. Kobashigawa, K. Suzuki, S. Yamashita, Ionizing radiation accelerates Drp1- dependent mitochondrial fission, which involves delayed mitochondrial reactive oxygen species production in normal human fibroblast-like cells, Biochem. Biophys. Res. Commun. 414 (2011) 795-800.

[109] J.P. Munoz, S.k. Ivanova, J. Sanchez-Wandelmer, P. Martinez-Cristòbal, E. Noguera, A. Sancho, et al., Mfn2 modulates the UPR and mitochondrial function via repression of PERK, EMBO J. 32 (2013) 2348-2361.

[110] P.H. Willems, R. Rossignol, C.E. Dieteren, M.P. Murphy, W.J. Koopman, Redox homeostasis and mitochondrial dynamics, Cell Metab. 22 (2015) 207-218.

[111] O.Y. Pletjushkina, K. Lyamzaev, E. Popova, O. Nepryakhina, O.Y. Ivanova, L. Domnina, et al., Effect of oxidative stress on dynamics of mitochondrial reticulum, Biochim. Biophys. Acta (BBA)—Bioenerg. 1757 (2006) 518-524.

[112] G.-Y. Liou, P. Storz, Reactive oxygen species in cancer, Free Radic. Res. 44 (2010) 479-496.

[113] A. Chatterjee, E. Mambo, D. Sidransky, Mitochondrial DNA mutations in human cancer, Oncogene 25 (34) (2006) 4663-4674 x.

[114] G.M. DeNicola, F.A. Karreth, T.J. Humpton, A. Gopinathan, C. Wei, K. Frese, et al., Oncogene-induced Nrf2 transcription promotes ROS detoxification and tumorigenesis, Nature 475 (2011) 106-109.

[115] E. Piskounova, M. Agathocleous, M.M. Murphy, Z. Hu, S.E. Huddlestun, Z. Zhao, et al., Oxidative stress inhibits distant metastasis by human melanoma cells, Nature 527 (7577) (2015) 186-191.

[116] S. Parangi, M. O'Reilly, G. Christofori, L. Holmgren, J. Grosfeld, J. Folkman, et al., Antiangiogenic therapy of transgenic mice impairs de novo tumor growth, Proc. Natl. Acad. Sci. U. S. A. 93 (1996) 2002-2007.

[117] C. Xia, Q. Meng, L.-Z. Liu, Y. Rojanasakul, X.-R. Wang, B.-H. Jiang, Reactive oxygen species regulate angiogenesis and tumor growth through vascular endothelial growth factor, Cancer Res. 67 (2007) 10823-10830.

[118] Y.-W. Kim, T.V. Byzova, Oxidative stress in angiogenesis and vascular disease, Blood 123 (2014) 625-631.

[119] S.-N. Jung, W.K. Yang, J. Kim, H.S. Kim, E.J. Kim, H. Yun, et al., Reactive oxygen species stabilize hypoxia-inducible factor- 1 alpha protein and stimulate transcriptional activity via AMP-activated protein kinase in DU145 human prostate cancer cells, Carcinogenesis 29 (2008) 713-721.

[120] N. Nishida, H. Yano, T. Nishida, T. Kamura, M. Kojiro, Angiogenesis in cancer, Vasc. Health Risk Manage. 2 (2006) 213.

[121] J.-w. Kim, I. Tchernyshyov, G.L. Semenza, C.V. Dang, HIF-1-mediated expression of pyruvate dehydrogenase kinase: a metabolic switch required for cellular adaptation to hypoxia, Cell Metab. 3 (2006) 177-185.

[122] D. Huang, T. Li, X. Li, L. Zhang, L. Sun, X. He, et al., HIF-1-mediated suppression of acyl-CoA dehydrogenases and fatty acid oxidation is critical for cancer progression, Cell Rep. 8 (2014) 1930-1942.

[123] G.L. Semenza, Hypoxia-inducible factors: coupling glucose metabolism and redox regulation with induction of the breast cancer stem cell phenotype, EMBO J. (2016) e201695204.

[124] G. Twig, A. Elorza, A.J. Molina, H. Mohamed, J.D. Wikstrom, G. Walzer, et al., Fission and selective fusion govern mitochondrial segregation and elimination by autophagy, EMBO J. 27 (2008) 433-446.

[125] A.W. Greene, K. Grenier, M.A. Aguileta, S. Muise, R. Farazifard, M.E. Haque, et al., Mitochondrial processing peptidase regulates PINK1 processing, import and Parkin recruitment, EMBO Rep. 13 (2012) 378-385.

[126] L.A. Kane, M. Lazarou, A.I. Fogel, Y. Li, K. Yamano, S.A. Sarraf, et al., PINK1 phosphorylates ubiquitin to activate Parkin E3 ubiquitin ligase activity, J. Cell Biol. (2014), http://dx.doi.org/10.1083/jcb.201402104.

[127] M. Lazarou, D.A. Sliter, L.A. Kane, S.A. Sarraf, C. Wang, J.L. Burman, et al., The ubiquitin kinase PINK1 recruits autophagy receptors to induce mitophagy, Nature 524 (2015) 309-314.

[128] F. Strappazzon, F. Nazio, M. Corrado, V. Cianfanelli, A. Romagnoli, G.M. Fimia, et al., AMBRA1 is able to induce mitophagy via LC3 binding, regardless of PARKIN and p62/SQSTM1, Cell Death Differ. 22 (2015) 419-432.

[129] W. Wu, W. Tian, Z. Hu, G. Chen, L. Huang, W. Li, et al., ULK1 translocates to mitochondria and phosphorylates FUNDC1 to regulate mitophagy, EMBO Rep. (2014) e201438501.

[130] C.T. Chu, J. Ji, R.K. Dagda, J.F. Jiang, Y.Y. Tyurina, A.A. Kapralov, et al., Cardiolipin externalization to the outer mitochondrial membrane acts as an elimination signal for mitophagy in neuronal cells, Nat. Cell Biol. 15 (2013) 1197-1205.

[131] L. Liu, K. Sakakibara, Q. Chen, K. Okamoto, Receptor-mediated mitophagy in yeast and mammalian systems, Cell Res. 24 (2014) 787-795.

[132] J. Zhang, P.A. Ney, Role of BNIP3 and NIX in cell death autophagy, and mitophagy, Cell Death Differ. 16 (2009) 939-946.

[133] A.M. Pickrell, R.J. Youle, The roles of PINK1, parkin, and mitochondrial fidelity in Parkinson's disease, Neuron 85 (2015) 257-273.

[134] X. Wang, D. Winter, G. Ashrafi, J. Schlehe, Y.L. Wong, D. Selkoe, et al., PINK1 and Parkin target Miro for phosphorylation and degradation to arrest mitochondrial motility, Cell 147 (2011) 893-906.

[135] E.Q. Toyama, S.b. Herzig, J. Courchet, T.L. Lewis, O.C. Losòn, K. Hellberg, et al., AMP-activated protein kinase mediates mitochondrial fission in response to energy stress, Science 351 (2016) 275-281.

[136] M.A. Bin-Umer, J.E. McLaughlin, M.S. Butterly, S. McCormick, N.E. Tumer, Elimination of damaged mitochondria through mitophagy reduces mitochondrial oxidative stress and increases tolerance to trichothecenes, Proc. Natl. Acad. Sci. U. S. A. 111 (2014) 11798-11803.

[137] Y. Kurihara, T. Kanki, Y. Aoki, Y. Hirota, T. Saigusa, T. Uchiumi, et al., Mitophagy plays an essential role in reducing mitochondrial production of reactive oxygen species and mutation of mitochondrial DNA by maintaining mitochondrial quantity and quality in yeast, J. Biol. Chem. 287 (2012) 3265-3272. 
[138] X. Wei, Y. Qi, X. Zhang, Q. Qiu, X. Gu, C. Tao, et al., Cadmium induces mitophagy through ROS-mediated PINK1/Parkin pathway, Toxicol. Mech. Methods 24 (2014) 504-511.

[139] A.P. Joselin, S.J. Hewitt, S.M. Callaghan, R.H. Kim, Y.-H. Chung, T.W. Mak, et al., ROS-dependent regulation of Parkin and DJ-1 localization during oxidative stress in neurons, Hum. Mol. Genet. (2012) dds325.

[140] S. Matsuda, A. Nakanishi, A. Minami, Y. Wada, Y. Kitagishi, Functions and characteristics of PINK1 and Parkin in cancer, Front. Biosci. (Landmark Edition) 20 (2014) 491-501.

[141] T.J. Pugh, O. Morozova, E.F. Attiyeh, S. Asgharzadeh, J.S. Wei, D. Auclair, et al., The genetic landscape of high-risk neuroblastoma, Nat. Genet. 45 (2013) 279-284.

[142] E. White, The role for autophagy in cancer, J. Clin. Invest. 125 (2015) 42-46.

[143] V. Cianfanelli, C. Fuoco, M. Lorente, M. Salazar, F. Quondamatteo, P.F. Gherardini, et al., AMBRA1 links autophagy to cell proliferation and tumorigenesis by promoting c-Myc dephosphorylation and degradation, Nat. Cell Biol. 17 (2015) 20-30.

[144] Q. Huang, L. Zhan, H. Cao, J. Li, Y. Lyu, X. Guo, et al., Increased mitochondria fission promotes autophagy and hepatocellular carcinoma cell survival through the ROS-modulated coordinated regulation of the NFKB and TP53 pathways, Autophagy (2016) 1-16.

[145] J.Y. Guo, H.-Y. Chen, R. Mathew, J. Fan, A.M. Strohecker, G. Karsli-Uzunbas, et al., Activated Ras requires autophagy to maintain oxidative metabolism and tumorigenesis, Genes. Dev. 25 (2011) 460-470.

[146] D. Senft, A.R. Ze'ev, Regulators of mitochondrial dynamics in cancer, Curr. Opin. Cell Biol. 39 (2016) 43-52.

[147] J.A. Kashatus, A. Nascimento, L.J. Myers, A. Sher, F.L. Byrne, K.L. Hoehn, et al., Erk2 phosphorylation of Drp1 promotes mitochondrial fission and MAPK-driven tumor growth, Mol. Cell 57 (2015) 537-551.

[148] M.N. Serasinghe, S.Y. Wieder, T.T. Renault, R. Elkholi, J.J. Asciolla, J.L. Yao, et al., Mitochondrial division is requisite to RAS-induced transformation and targeted by oncogenic MAPK pathway inhibitors, Mol. Cell 57 (2015) 521-536.

[149] Y.-Y. Wan, J.-F. Zhang, Z.-J. Yang, L.-P. Jiang, Y.F. Wei, Q.-N. Lai, et al., Involvement of Drp1 in hypoxia-induced migration of human glioblastoma U251 cells, Oncol. Rep. 32 (2014) 619-626.

[150] G.-E. Zhang, H.-L. Jin, X.-K. Lin, C. Chen, X.-S. Liu, Q. Zhang, et al., Anti-tumor effects of Mfn2 in gastric cancer, Int. J. Mol. Sci. 14 (2013) 13005-13021.

[151] W. Wang, Q. Xie, X. Zhou, J. Yao, X. Zhu, P. Huang, et al., Mitofusin-2 triggers mitochondria $\mathrm{Ca} 2+$ influx from the endoplasmic reticulum to induce apoptosis in hepatocellular carcinoma cells, Cancer Lett. 358 (2015) 47-58.

[152] X. Li, F. Wang, Z. Wu, J. Lin, W. Lan, J. Lin, MicroRNA-19b targets Mfn1 to inhibit Mfn1-induced apoptosis in osteosarcoma cells, Neoplasma 61 (2013) 265-273.

[153] F. Poutèn, K. Jirstrom, M. Uhlen, The human protein atlas - a tool for pathology, J. Pathol. 216 (4) (2008) 387-393.

[154] A. Sehrawat, C.S. Croix, C.J. Baty, S. Watkins, D. Tailor, R.P. Singh, et al., Inhibition of mitochondrial fusion is an early and critical event in breast cancer cell apoptosis by dietary chemopreventative benzyl isothiocyanate, Mitochondrion 30 (2016) 67-77.

[155] Y. Kubohara, Effects of differentiation-inducing factors of Dictyostelium dis coideum on human leukemia K562 cells: DIF-3 is the most potent anti-leukemic agent, Eur. J. Pharmacol. 381 (1) (1999) 57-62.

[156] A. Dubois, C. Ginet, N. Furstoss, A. Belaid, M.A. Hamouda, W. El Manaa, et al., Differentiation inducing factor 3 mediates its anti-leukemic effect through ROSdependent DRP1-mediated mitochondrial fission and induction of caspase-independent cell death, Oncotarget 7 (18) (2016) 26120-26136.

[157] Y. Jia, L. Zhou, C. Tian, Y. Shi, C. Wang, Z. Tong, Dynamin-related protein 1 is involved in micheliolide-induced breast cancer cell death, OncoTargets Ther. 8 (2015) 3371.

[158] A. Cassidy-Stone, J.E. Chipuk, E. Ingerman, C. Song, C. Yoo, T. Kuwana, et al., Chemical inhibition of the mitochondrial division dynamin reveals its role in Bax/ Bak-dependent mitochondrial outer membrane permeabilization, Dev. Cell 14 (2008) 193-204.

[159] J. Wang, J. Li, L. Santana-Santos, M. Shuda, R.W. Sobol, B. Van Houten, et al., A novel strategy for targeted killing of tumor cells: induction of multipolar acentrosomal mitotic spindles with a quinazolinone derivative mdivi-1, Mol. Oncol. 9 (2015) 488-502.

[160] A.A. Rosdah, J.K. Holien, L. Delbridge, G.J. Dusting, S.Y. Lim, Mitochondrial fission-a drug target for cytoprotection or cytodestruction? Pharmacol. Res. Perspect. 4 (2016).

[161] Q. Xie, Q. Wu, C.M. Horbinski, W.A. Flavahan, K. Yang, W. Zhou, et al., Mitochondrial control by DRP1 in brain tumor initiating cells, Nat. Neurosci. 18 (2015) 501-510.

[162] M. Akita, M. Suzuki-Karasaki, K. Fujiwara, C. Nakagawa, M. Soma, Y. Yoshida, et al., Mitochondrial division inhibitor-1 induces mitochondrial hyperfusion and sensitizes human cancer cells to TRAIL-induced apoptosis, Int. J. Oncol. 45 (2014) 1901-1912.

[163] J. Wang, K. Hansen, R. Edwards, B. Van Houten, W. Qian, Mitochondrial division inhibitor 1 (mdivi-1) enhances death receptor-mediated apoptosis in humar ovarian cancer cells, Biochem. Biophys. Res. Commun. 456 (2015) 7-12.

[164] W. Qian, J. Wang, V. Roginskaya, L.A. McDermott, R.P. Edwards, D.B. Stolz, et al., Novel combination of mitochondrial division inhibitor 1 (mdivi-1) and platinum agents produces synergistic pro-apoptotic effect in drug resistant tumor cells, Oncotarget 5 (2014) 4180.

[165] X.-J. Han, Z.-J. Yang, L.-P. Jiang, Y.-F. Wei, M.-F. Liao, Y. Qian, et al., Mitochondrial dynamics regulates hypoxia-induced migration and antineoplastic activity of cisplatin in breast cancer cells, Int. J. Oncol. 46 (2015) 691-700.

[166] Y. Kushnareva, A.Y. Andreyev, T. Kuwana, D.D. Newmeyer, Bax activation initiates the assembly of a multimeric catalyst that facilitates Bax pore formation in mitochondrial outer membranes, PLoS Biol. 10 (2012) e1001394.

[167] E.A. Bordt, P. Clerc, B.A. Roelofs, A.J. Saladino, L.s. Tretter, V. Adam-Vizi, et al., The putative Drp1 inhibitor mdivi-1 is a reversible mitochondrial complex I inhibitor that modulates reactive oxygen species, Dev. Cell 40 (2017) 583.e6-594.e6.

[168] X. Qi, N. Qvit, Y.-C. Su, D. Mochly-Rosen, A novel Drp1 inhibitor diminishes aberrant mitochondrial fission and neurotoxicity, J. Cell Sci. 126 (2013) 789-802.

[169] D. Wang, J. Wang, G. Bonamy, S. Meeusen, R.G. Brusch, C. Turk, et al., A small molecule promotes mitochondrial fusion in mammalian cells, Angew. Chem. Int. Ed. 51 (2012) 9302-9305.

[170] T. Blankenstein, P.G. Coulie, E. Gilboa, E.M. Jaffee, The determinants of tumour immunogenicity, Nat. Rev. Cancer 12 (2012) 307-313.

[171] L. Aymeric, L. Apetoh, F.o. Ghiringhelli, A. Tesniere, I. Martins, G. Kroemer, et al., Tumor cell death and ATP release prime dendritic cells and efficient anticancer immunity, Cancer Res. 70 (2010) 855-858.

[172] J. Fucikova, I. Moserova, L. Urbanova, L. Bezu, O. Kepp, I. Cremer, et al., Prognostic and predictive value of DAMPs and DAMP-associated processes in cancer, Front. Immunol. 6 (2015).

[173] L. Galluzzi, A. Buqué, O. Kepp, L. Zitvogel, G. Kroemer, Immunogenic cell death in cancer and infectious disease, Nat. Rev. Immunol. 17 (2016) 97-111.

[174] H. Guo, J.B. Callaway, J.P. Ting, Inflammasomes: mechanism of action, role in disease, and therapeutics, Nat. Med. 21 (2015) 677-687.

[175] R. Zhou, A.S. Yazdi, P. Menu, J.r. Tschopp, A role for mitochondria in NLRP3 inflammasome activation, Nature 469 (2011) 221-225.

[176] J.-H. Won, S. Park, S. Hong, S. Son, J.-W. Yu, Rotenone-induced impairment of mitochondrial electron transport chain confers a selective priming signal for NLRP3 inflammasome activation, J. Biol. Chem. 290 (2015) 27425-27437.

[177] S.S. Iyer, Q. He, J.R. Janczy, E.I. Elliott, Z. Zhong, A.K. Olivier, et al., Mitochondrial cardiolipin is required for Nlrp3 inflammasome activation, Immunity 39 (2013) 311-323.

[178] I.C. Allen, M.A. Scull, C.B. Moore, E.K. Holl, E. McElvania-TeKippe, D.J. Taxman, et al., The NLRP3 inflammasome mediates in vivo innate immunity to influenza A virus through recognition of viral RNA, Immunity 30 (2009) 556-565.

[179] A. Dashdorj, K.R. Jyothi, S. Lim, A. Jo, M.N. Nguyen, J. Ha, et al., Mitochondriatargeted antioxidant MitoQ ameliorates experimental mouse colitis by suppressing NLRP3 inflammasome-mediated inflammatory cytokines, BMC Med. 11 (2013) 178.

[180] C. Juliana, T. Fernandes-Alnemri, S. Kang, A. Farias, F. Qin, E.S. Alnemri, Nontranscriptional priming and deubiquitination regulate NLRP3 inflammasome activation, J. Biol. Chem. 287 (2012) 36617-36622.

[181] T. Ichinohe, T. Yamazaki, T. Koshiba, Y. Yanagi, Mitochondrial protein mitofusin 2 is required for NLRP3 inflammasome activation after RNA virus infection, Proc. Natl. Acad. Sci. U. S. A. 110 (2013) 17963-17968.

[182] T. Koshiba, N. Bashiruddin, S. Kawabata, Mitochondria and antiviral innate immunity, Int. J. Biochem. Mol. Biol. 2 (2011) 257-262.

[183] C. Castanier, D. Garcin, A. Vazquez, D. Arnoult, Mitochondrial dynamics regulate the RIG-I-like receptor antiviral pathway, EMBO Rep. 11 (2010) 133-138.

[184] S. Park, J.-H. Won, I. Hwang, S. Hong, H.K. Lee, J.-W. Yu, Defective mitochondrial fission augments NLRP3 inflammasome activation, Sci. Rep. 5 (2015) 15489.

[185] P. Liu, Q. Xie, T. Wei, Y. Chen, H. Chen, W. Shen, Activation of the NLRP3 inflammasome induces vascular dysfunction in obese OLETF rats, Biochem. Biophys. Res. Commun. 468 (2015) 319-325.

[186] X. Wang, W. Jiang, Y. Yan, T. Gong, J. Han, Z. Tian, et al., RNA viruses promote activation of the NLRP3 inflammasome through a RIP1-RIP3-DRP1 signaling pathway, Nat. Immunol. 15 (2014) 1126-1133.

[187] J. Park, H. Choi, J.S. Min, S.J. Park, J.H. Kim, H.J. Park, et al., Mitochondrial dynamics modulate the expression of pro-inflammatory mediators in microglial cells, J. Neurochem. 127 (2013) 221-232.

[188] Y.J. Kang, B.-R. Bang, K.H. Han, L. Hong, E.-J. Shim, J. Ma, et al., Regulation of NKT cell-mediated immune responses to tumours and liver inflammation by mitochondrial PGAM5-Drp1 signalling, Nat. Commun. 6 (2015).

[189] J. Park, H. Choi, J.S. Min, S.J. Park, J.H. Kim, H.J. Park, et al., Mitochondrial dynamics modulate the expression of pro-inflammatory mediators in microglial cells, J. Neurochem. 127 (2013) 221-232.

[190] I. Waldhauer, A. Steinle, NK cells and cancer immunosurveillance, Oncogene 27 (2008) 5932-5943.

[191] A. Cerwenka, L.L. Lanier, Natural killer cell memory in infection, inflammation and cancer, Nat. Rev. Immunol. 16 (2016) 112-123.

[192] T.E. O'Sullivan, L.R. Johnson, H.H. Kang, J.C. Sun, BNIP3-and BNIP3L-mediated mitophagy promotes the generation of natural killer cell memory, Immunity 43 (2015) 331-342.

[193] E. Abarca-Rojano, S. Muniz-Hernàndez, M.M.B. Moreno-Altamirano, R. Mondragòn-Flores, F. Enriquez-Rincòn, F.J. Sànchez-Garcìa, Re-organization of mitochondria at the NK cell immune synapse, Immunol. Lett. 122 (2009) 18-25.

[194] N. Lee, L.R. Zakka, M.C. Mihm, T. Schatton, Tumour-infiltrating lymphocytes in melanoma prognosis and cancer immunotherapy, Pathology 48 (2016) 177-187.

[195] F. Noble, T. Mellows, L.H.M. Matthews, A.C. Bateman, S. Harris, T.J. Underwood, et al., Tumour infiltrating lymphocytes correlate with improved survival in patients with oesophageal adenocarcinoma, Cancer Immunol. Immunother. (2016) $1-12$.

[196] R. Wu, M.-A. Forget, J. Chacon, C. Bernatchez, C. Haymaker, J.Q. Chen, et al., Adoptive T-cell therapy using autologous tumor-infiltrating lymphocytes for metastatic melanoma: current status and future outlook, Cancer J. (Sudbury, Mass) 18 (2012) 160.

[197] C. Schwindling, A. Quintana, E. Krause, M. Hoth, Mitochondria positioning 
controls local calcium influx in T cells, J. Immunol. 184 (2010) 184-190.

[198] A. Quintana, C. Schwindling, A.S. Wenning, U. Becherer, J. Rettig, E.C. Schwarz, et al., $\mathrm{T}$ cell activation requires mitochondrial translocation to the immunological synapse, Proc. Natl. Acad. Sci. U. S. A. 104 (2007) 14418-14423.

[199] A. Quintana, M. Pasche, C. Junker, D. Al-Ansary, H. Rieger, C. Kummerow, et al., Calcium microdomains at the immunological synapse: how ORAI channels, mitochondria and calcium pumps generate local calcium signals for efficient T-cell activation, EMBO J. 30 (2011) 3895-3912.

[200] D. Roth, P.H. Krammer, K. Gulow, Dynamin related protein 1-dependent mitochondrial fission regulates oxidative signalling in T cells, FEBS Lett. 588 (9) (2014) 1749-1754.

[201] M.D. Buck, D. O'Sullivan, E.L. Pearce, T cell metabolism drives immunity, J. Exp. Med. 212 (9) (2015) 1345-1360.

[202] M.D. Buck, D. O'Sullivan, R.I.K. Geltink, J.D. Curtis, C.-H. Chang, D.E. Sanin, et al, Mitochondrial dynamics controls $\mathrm{T}$ cell fate through metabolic programming, Cell 166 (1) (2016) 63-76.

[203] E.L. Pearce, M.C. Poffenberger, C.-H. Chang, R.G. Jones, Fueling immunity: insights into metabolism and lymphocyte function, Science 342 (2013) 1242454

[204] G.J. van der Windt, B. Everts, C.-H. Chang, J.D. Curtis, T.C. Freitas, E. Amiel, et al., Mitochondrial respiratory capacity is a critical regulator of CD8 + T cell memory development, Immunity 36 (2012) 68-78.

[205] J. Jacobelli, M.E. Matthews, S. Chen, M.F. Krummel, Activated T cell trans-endothelial migration relies on myosin-IIA contractility for squeezing the cell nucleus through endothelial cell barriers, PLoS One 8 (2013) e75151.

[206] Y. Jiang, Y. Li, B. Zhu, T-cell exhaustion in the tumor microenvironment, Cell. Death. Dis. 6 (6) (2015) e1792.

[207] N.E. Scharping, A.V. Menk, R.S. Moreci, R.D. Whetstone, R.E. Dadey, S.C. Watkins, et al., The tumor microenvironment represses $\mathrm{T}$ cell mitochondrial biogenesis to drive intratumoral T cell metabolic insufficiency and dysfunction, Immunity 45 (2016) 374-388.
[208] C.-H. Chang, J. Qiu, D. O'Sullivan, M.D. Buck, T. Noguchi, J.D. Curtis, et al., Metabolic competition in the tumor microenvironment is a driver of cancer progression, Cell 162 (2015) 1229-1241.

[209] M. Liesa, O.S. Shirihai, Mitochondrial dynamics in the regulation of nutrient utilization and energy expenditure, Cell Metab. 17 (4) (2013) 491-506.

[210] R. Geiger, J.C. Rieckmann, T. Wolf, C. Basso, Y. Feng, T. Fuhrer, et al., L-Arginine modulates T cell metabolism and enhances survival and anti-tumor activity, Cell 167 (2016) 829e.13-842.e13.

[211] P. Fisicaro, V. Barili, B. Montanini, G. Acerbi, M. Ferracin, F. Guerrieri, et al., Targeting mitochondrial dysfunction can restore antiviral activity of exhausted HBV-specific CD8T cells in chronic hepatitis B, Nat. Med. 23 (2017) 327-336.

[212] K.E. Pauken, E.J. Wherry, Overcoming T cell exhaustion in infection and cancer, Trends Immunol. 36 (4) (2015) 265-276.

[213] M. Corrado, F.R. Mariotti, L. Trapani, L. Taraborrelli, F. Nazio, V. Cianfanelli, et al., Macroautophagy inhibition maintains fragmented mitochondria to foster T cell receptor-dependent apoptosis, EMBO J. (2016) e201593727.

[214] S. Maher, D. Toomey, C. Condron, D. Bouchier-Hayes, Activation-induced cell death: the controversial role of Fas and Fas ligand in immune privilege and tumour counterattack, Immunol. Cell Biol. 80 (2002) 131-137.

[215] M. Tabbekh, K. Franciszkiewicz, H. Haouas, Y. Lécluse, K. Benihoud, C. Raman, et al., Rescue of tumor-infiltrating lymphocytes from activation-induced cell death enhances the antitumor CTL response in CD5-deficient mice, J. Immunol. 187 (2011) 102-109.

[216] L.M. Coussens, Z. Werb, Inflammation and cancer, Nature 420 (2002) 860-867.

[217] S.F. Schoppmann, P. Birner, J. Stockl, R. Kalt, R. Ullrich, C. Caucig, et al., Tumor associated macrophages express lymphatic endothelial growth factors and are related to peritumoral lymphangiogenesis, Am. J. Pathol. 161 (2002) 947-956.

[218] B.H. Zinselmeyer, S. Heydari, C. Sacristàn, D. Nayak, M. Cammer, J. Herz, et al., PD-1 promotes immune exhaustion by inducing antiviral T cell motility paralysis, J. Exp. Med. 210 (2013) 757-774. 